\title{
Unified Topological Response Theory For Gapped and Gapless Free Fermions
}

\author{
Daniel Bulmash, Pavan Hosur, Shou-Cheng Zhang, and Xiao-Liang Qi \\ Department of Physics, Stanford University, Stanford, California 94305-4045, USA \\ (Received 7 November 2014; revised manuscript received 26 February 2015; published 26 May 2015)

\begin{abstract}
We derive a scheme for systematically characterizing the responses of gapped as well as gapless systems
\end{abstract} \\ of free fermions to electromagnetic and strain fields starting from a common parent theory. Using the fact \\ that position operators in the lowest Landau level of a quantum Hall state are canonically conjugate, we \\ consider a massive Dirac fermion in $2 n$ spatial dimensions under $n$ mutually orthogonal magnetic fields and \\ reinterpret physical space in the resulting zeroth Landau level as phase space in $n$ spatial dimensions. The \\ bulk topological responses of the parent Dirac fermion, given by a Chern-Simons theory, translate into \\ quantized insulator responses, while its edge anomalies characterize the response of gapless systems. \\ Moreover, various physically different responses are seen to be related by the interchange of position and \\ momentum variables. We derive many well-known responses and demonstrate the utility of our theory by \\ predicting spectral flow along dislocations in Weyl semimetals.
}

DOI: 10.1103/PhysRevX.5.021018

\section{INTRODUCTION}

Spurred by the discovery of topological insulators, topological phases have become a vital part of condensed-matter physics over the last decade [1-4]. Even in the absence of interactions, a wide variety of gapped topological phases of fermions are now known, ranging from the quantum Hall $[5,6]$ and the quantum spin Hall [7-13] insulators (among insulators) to the chiral $p$-wave superconductor [14,15] and the $B$ phase of Helium-3 $[16,17]$ (among superconducting phases). All these phases share some common features: As long as certain symmetry conditions are upheld, they have a bulk band structure that cannot be deformed into that of an atomic insulator-a trivial insulator, by definition-without closing the band gap along the way. Moreover, they all have robust surface states that mediate unusual transport immune to symmetryrespecting disorder. These features lead one to wonder whether all gapped phases of free fermions can be unified within a common mathematical framework.

Two different approaches have been developed to provide unified characterization of gapped phases of free fermions. In the topological band theory approach [18-20], homotopy theory and $K$ theory are applied to classify freefermion Hamiltonians in a given spatial dimension and symmetry class. The topological band theory provides a complete topological classification of free-fermion gapped states in all dimensions and all ten Altland-Zirnbauer symmetry classes [21]. However, it does not directly

Published by the American Physical Society under the terms of the Creative Commons Attribution 3.0 License. Further distribution of this work must maintain attribution to the author(s) and the published article's title, journal citation, and DOI.
Subject Areas: Condensed Matter Physics

describe physical properties of the topological states. In comparison, the topological response theory approach [22-26] describes topological phases by topological terms in their response to external gauge fields and gravitational fields. The advantage of this approach is that the topological phases are characterized by physically observable topological effects, so the robustness of the topological phase is explicit and more general than in the topological band theory. Since it is insensitive to details of the microscopic Hamiltonian, a response-theory-based classification scheme can be further extended to strongly interacting systems [27].

Recently, the advent of Weyl semimetals (WSMs) has triggered interest in gapless topological phases of free fermions [28-34]. These phases are topological in the sense that they cannot be gapped out perturbatively as long as momentum and charge are conserved. In this regard, ordinary metals are also topological since their Fermi surfaces are robust in the absence of instabilities towards density waves or superconductivity. Additionally, gapless topological phases may have nontrivial surface states such as Fermi arcs [28,35-37] and flat bands [38]. Teo and Kane [39] applied homotopy arguments to classify topological defects such as vortices and dislocations in gapped phases; Matsuura et al. [40] used an analogous prescription to classify gapless phases by observing that gapless regions in momentum space such as Fermi surfaces and Dirac nodes can be viewed as topological defects in momentum space in a gapped system. Thus, a common mathematical formalism to describe the Bloch Hamiltonians of gapless phases was derived.

Unlike their gapped counterparts, however, it is not clear whether the response theories of gapless phases are amenable to a unified description. For gapped systems, the path from the Hamiltonian to the response theory is 
conceptually straightforward: The fermions are coupled to gauge fields and integrated out to get the low-energy effective field theory, which describes the topological response properties. In contrast, the low-energy theory of gapless phases contains fermions as well as gauge fields and is distinct from the response theory which contains only gauge fields. Thus, it is not obvious how the topological properties of the Hamiltonian affect the response. The response depends on system details, in general, and therefore, recognizing its universal features and then unifying the responses of various gapless phases is a nontrivial task. A few cases of topological response properties of gapless fermions have been studied. One example is the intrinsic anomalous Hall effect of a twodimensional Fermi gas [41-45]. A generalization of this effect in three-dimensional doped topological insulators has been discussed [46]. Another example is the topological response of Weyl semimetals, which has been described in the form of the axial anomaly $[34,47-60]$. This refers to the apparent charge conservation violation that occurs for each Weyl fermion branch in the presence of parallel electric and magnetic fields, although the net charge of the system must still be conserved. Recently, these ideas were generalized to find the topological responses of point Fermi surfaces in arbitrary dimensions [61]. The dc conductivity of metals has also been proposed to be related to a phase-space topological quantity [62]. However, a general theory that describes the topological properties of gapless fermions in a unified framework has not been developed yet.

In this work, we achieve the above goals for free fermions: We show that gapless systems have universal features, independent of system details, and derive a unified description of their response. Remarkably, this description also captures the response of gapped systems. In particular, the response of gapped phases arises from the bulk response of a certain parent topological phase, while the universal features of the gapless phases correspond to its edge anomalies. We elucidate this idea below.

The backbone of our construction is a mapping from $n$-dimensional gapped or gapless systems to a gapped quantum Hall $(\mathrm{QH})$ system that lives in $2 n$-dimensional phase space. Such a phase-space system has both bulk responses, given by a $2 n$-dimensional Chern-Simons (CS) theory, and boundary (axial) anomalies. We identify the bulk responses with topological responses of insulators. However, the key insight that allows us to include gapless systems is to identify a Fermi surface in real space with a phase-space boundary in the momentum directions. Likewise, real-space excitations near the Fermi surface are identified with the gapless edge excitations in phase space. The universal features of the response of gapless systems are thus the anomalies associated with these phase-space edges.

There is an important technical point required in order to bestow the $2 n$-dimensional QH system with the interpretation of phase space. Specifically, we must choose the QH system to consist of a massive Dirac fermion under $n$ uniform magnetic fields of strength $B_{0}$ in $n$ orthogonal planes, and then project to the zeroth Landau level (ZLL) of the total field. In this case, the projected operators for pairs of dimensions acquire the usual canonical commutation relations that relate ordinary real and momentum space (up to an overall factor of $B_{0}$ ). This result allows us to interpret the ZLL of the $2 n$-dimensional QH system as phase space for the $n$-dimensional physical space. We interpret additional perturbations in the phase-space gauge fields as physical quantities such as the $n$-dimensional system's electromagnetic (EM) field, strain field, Berry curvatures, and Hamiltonian. Topological defects, such as monopoles, in the phase-space gauge fields allow us to generalize to systems with dislocations and with point Fermi surfaces such as graphene and Weyl semimetals. These ideas are summarized in Table I.

This construction enables us to systematically enumerate all possible intrinsic, topological responses to electromagnetic and strain fields in the dc limit in any given dimension. One simply has to write down the ChernSimons action in phase space, vary it with respect to each gauge field, and consider each boundary to obtain all the bulk, boundary, and gapless responses in real space. Following this procedure, we show carefully that screw dislocations in Weyl semimetals trap chiral modes which are well localized around the dislocation at momentum values away from the Weyl nodes. A related but different effect has been studied previously [63]. However, our framework provides a unified and natural description of this effect and other topological effects.

It is crucial that the $2 n$-dimensional system be gapped even in the absence of the background magnetic fields of strength $B_{0}$. This ensures that its response theory contains terms depending on $B_{0}$ in addition to fluctuations in the gauge fields. In $n$ dimensions, we will see that the $B_{0}$-dependent terms translate into quasi-lower-dimensional responses, such as the polarization of a system of coupled chains as measured along the chains. If the $2 n$-dimensional

TABLE I. Dictionary for interpreting phase-space quantities in real space.

\begin{tabular}{|c|c|}
\hline Phase space & Real space \\
\hline Bulk responses & Quantized insulator responses \\
\hline $\begin{array}{l}\text { Anomalies from momentum } \\
\text { direction edges }\end{array}$ & Gapless response \\
\hline $\begin{array}{l}\text { Anomalies from real } \\
\text { direction edges }\end{array}$ & Real edge anomalies \\
\hline Gauge-field strength & $\begin{array}{c}\text { EM-field strength } / k \text {-space } \\
\text { Berry curvature/strain }\end{array}$ \\
\hline Monopole in gauge field & $\begin{array}{c}\text { Magnetic monopole/Weyl } \\
\text { node/dislocation }\end{array}$ \\
\hline
\end{tabular}


system is gapless in the absence of the background fields, such responses will be missed by the unified theory.

A caveat is that our construction does not capture responses to spatial, momental, and temporal variations in the field strengths, such as the gyrotropic effect which is an electric response to a spatially varying electric field. Note that the regular Maxwell response, given by $j^{\mu}=\partial_{\nu} F^{\nu \mu}$, is a response to a variation in the field strength. Another caveat is that in phase-space dimensions equal to 4 and above, the Maxwell term in the action is equally relevant to or more relevant than the Chern-Simons term and hence will, in general, dominate the dc response. However, our central objective is to demonstrate that there exists a theory that unifies the responses of gapped and gapless systems, namely, the phase-space Chern-Simons theory.

The rest of this paper is structured as follows. In Sec. II, we review the key property of the ZLL, which provides the physical justification for our construction. In Sec. III, we explain the interpretation of the gauge fields in our mapping and give an example illustrating the validity of the CS theory. In Sec. IV, we write down an explicit model with a CS response theory and show the precise way in which it behaves as the phase-space response theory of a lowerdimensional model. In Secs. V and VI, we explain the responses and anomalies (respectively) that come from the CS theory in various dimensions, applying our framework to describe spectral flow in Weyl semimetals with dislocations. Finally, in Sec. VII, we summarize our work and suggest extensions of our theory to more nontrivial systems.

\section{REVIEW OF THE ALGEBRA OF THE ZEROTH LANDAU LEVEL}

One of the key features that we use in the intuition for our approach is the fact that projecting position operators to the ZLL yields nonzero commutators between those operators. We now review this fact, for concreteness as well as for later convenience, for the case of Dirac electrons in a uniform magnetic field in two spatial dimensions in Landau gauge. Although we consider the ZLL of Dirac electrons here, the noncommutativity of position operators is simply a consequence of minimal coupling and Landau quantization of cyclotron orbits and hence is true for other dispersions as well as for other Landau levels for a Dirac dispersion.

Consider a 2D massive Dirac Hamiltonian in a uniform magnetic field,

$$
H=\left(p_{x}-e B y\right) \sigma_{x}+p_{y} \sigma_{y}+m \sigma_{z} .
$$

Here, $\sigma_{i}$ are the Pauli matrices. We have set the Fermi velocity to unity, written the electron charge as $-e$, and chosen the Landau gauge $\mathbf{A}=-B y \hat{\mathbf{x}}$ with $B>0$ for definiteness. Note that $p_{x}$ commutes with the
Hamiltonian, so we may replace it by its eigenvalue. We can define an annihilation operator $a=\left(p_{x}-e B y-i p_{y}\right) /$ $\sqrt{e B}$, which has $\left[a, a^{\dagger}\right]=1$, and the Hamiltonian becomes

$$
H=\left(\begin{array}{cc}
m & \sqrt{2 e B} a \\
\sqrt{2 e B} a^{\dagger} & -m
\end{array}\right) .
$$

It is straightforward to show that the eigenstates are labeled by an eigenvalue $n \geq 0$ of the number operator $a^{\dagger} a$, with dispersion $\pm \sqrt{2 e B n+m^{2}}$ for $n \neq 0$. For $n=0$, the eigenvalue is $-m$, the spin state is $\left(\begin{array}{l}0 \\ 1\end{array}\right)$, and the state is annihilated by $a$. As expected, the kinetic energy is quenched and the spectrum becomes discrete, highly degenerate Landau levels.

Let $\left|k_{x}\right\rangle$ be the state in the ZLL with $p_{x}$ eigenvalue $k_{x}$. Then, the projection operator to the ZLL is

$$
P=\int d k_{x} \frac{L}{2 \pi}\left|k_{x}\right\rangle\left\langle k_{x}\right|,
$$

with $L$ the system length in the $x$ direction. Writing $y=\left(\left(p_{x} / \sqrt{e B}\right)-\left(a+a^{\dagger}\right)\right) / \sqrt{e B}$, the projected $y$ operator becomes

$$
P y P=\int d k_{x} \frac{L}{2 \pi} \frac{k_{x}}{e B}\left|k_{x}\right\rangle\left\langle k_{x}\right|,
$$

where we have used the fact that $a$ and $a^{\dagger}$ describe interLandau-level processes and thus vanish under projection onto the ZLL. Next, using the fact that $\left|k_{x}\right\rangle$ is an eigenstate of $p_{x}$, we find

$$
P x P=\int d k_{x} \frac{L}{2 \pi} i \partial_{k_{x}}\left|k_{x}\right\rangle\left\langle k_{x}\right| .
$$

The commutator can then be easily computed as

$$
[P x P, P y P]=\frac{i}{e B} .
$$

Hence, if we absorb the factor of $e B$ into $y$, then $P x P$ and $P y P$ have the correct commutator structure for us to imbue them with the interpretation of the position and momentum operators, respectively, of a 1D system. This interpretation is the primary physical motivation for the construction that follows. As mentioned earlier, other dispersions will also result in commutation relations similar to Eq. (6) and thus imbue $x$ and $y$ with interpretations of position and momentum of a 1D system. However, a massive Dirac dispersion is ideal for deriving the unified response theory because it does not miss any quasi-lower-dimensional responses, as mentioned earlier and detailed later.

In higher dimensions, the Dirac model is $H=\sum_{i}\left(p_{i}+e A_{i}\right) \Gamma_{i}$, where the $\Gamma_{i}$ are anticommuting 
elements of the Clifford algebra of $2 n \times 2 n$ matrices. If we apply constant magnetic fields $F_{i j}$ for disjoint pairs $(i, j)$ of coordinates, we can form an annihilation operator for each such pair. Annihilation operators from different pairs commute, and the analysis above carries through so that the position operators within each pair no longer commute after projection.

\section{PHASE-SPACE CHERN-SIMONS THEORY}

The key idea of our construction is to represent a (possibly gapless) $n$-dimensional system by a gapped $2 n$-dimensional phase-space system, specifically a massive Dirac model coupled to a gauge field. As we just showed, we can interpret a $2 n$-dimensional system as living in phase space by adding background magnetic fields between disjoint pairs of spatial directions and projecting to the ZLL. Moreover, since the phase-space system is gapped, we can immediately write down a response theory for it, the topological part of which can be proved to be a CS theory $[22,64-66]$. Note that in a CS theory, real- and momentumspace gauge fields enter the action in similar ways, analogous to our idea of treating position and momentum on the same footing in phase space.

Before proceeding, we fix some notation and conventions. We will always use the Einstein summation convention where repeated indices are summed. Phase-space coordinates will be labeled by $x, y, z$ and $\bar{x}, \bar{y}, \bar{z}$. After projection, $x, y, z$ will be interpreted as the corresponding real-space coordinates, while $\bar{x}, \bar{y}, \bar{z}$ will be interpreted as momentum-space coordinates $k_{x}, k_{y}, k_{z}$, respectively. In phase space, we will refer to the $U(1)$ background gauge field that generates the real-space commutator structure as $A_{\mu}$, with its nonzero field strengths being $F_{i \bar{i}}=B_{0}$ for $i=(x, y, z)$. We denote all other contributions to the gauge field by $a_{\mu}$ and the total gauge field by $\mathcal{A}_{\mu}=A_{\mu}+a_{\mu}$. Likewise, we write $f_{\mu \nu}$ and $\mathcal{F}_{\mu \nu}=F_{\mu \nu}+f_{\mu \nu}$ for the nonbackground and total field strengths, respectively. The (non-Abelian) field strengths are, as usual, defined by $f_{\mu \nu}=\partial_{\mu} a_{\nu}-\partial_{\nu} a_{\mu}-\left[a_{\mu}, a_{\nu}\right]$.

We will also abbreviate the CS Lagrangian by $\epsilon a \partial a \equiv \epsilon^{\mu \nu \sigma} a_{\mu} \partial_{\nu} a_{\sigma}$, where $\epsilon$ is the totally antisymmetric Levi-Civita tensor, with an analogous abbreviation for higher-dimensional CS terms. Finally, we set $e=\hbar=1$ and also assume that $\sqrt{B_{0}} \sim 1 / l_{B}$ is very large compared to all other wave numbers in the problem.

\section{A. Interpretation of phase-space gauge fields}

Our prescription is that the nonbackground contributions $a_{\mu}$ to the phase-space gauge field should be interpreted as the Berry connection for the lower-dimensional system:

$$
a_{\mu}^{\alpha \beta}=i\left\langle u_{k \alpha}\left|\partial_{\mu}\right| u_{k \beta}\right\rangle,
$$

where $\left|u_{k \alpha}\right\rangle$ is the (local) Bloch wave function at momentum $k$ for the $\alpha$ band. Here, $\partial_{\bar{x}, \bar{y}, \bar{z}}$ should be interpreted as $B_{0} \partial_{k_{x}, k_{y}, k_{z}}$. We can think of the physical EM vector potential as a Berry connection, which means that it is included in the real-space components of $a$.

As such, we will often use the following heuristic interpretations in order to more clearly see the physics: $-a_{t}$ is the lower-dimensional band Hamiltonian plus the physical EM scalar potential, $a_{x, y, z}$ is the physical EM vector potential, and $a_{\bar{x}, \bar{y}, \bar{z}}$ is the momentum-space Berry connection. The field strengths that do not mix real and momentum space then have natural interpretations as the physical EM field strengths and Berry curvatures.

The physical interpretation of "mixed" field strengths such as $f_{x \bar{y}}$ (in four- or higher-dimensional phase space) is less obvious. Here, we present two ways to think about them. First, consider a gauge where $\partial_{\bar{y}} a_{x}=0$. We find that

$$
\int d \bar{y} f_{x \bar{y}}=\partial_{x} \int d \bar{y} a_{\bar{y}}=2 \pi \partial_{x} P_{y}
$$

where $P_{y}$ is the one-dimensional polarization of the system [67]. A spatially varying polarization can be thought of as strain of the electron wave function, which can come from either mechanical strain or some other spatial variation of the parameters entering the band structure.

To make the connection of $f_{x \bar{y}}$ to mechanical strain more explicit, we change the gauge to set $\partial_{x} a_{\bar{y}}=0$. An intuitive way to think about a nonzero $f_{x \bar{y}}$ in this gauge is in terms of dislocations. In particular, adiabatically moving a particle around a real-space dislocation leads to a translation, but if the particle can locally be treated as a Bloch wave, then that translation is equivalent to the accumulation of a phase. This (Berry) phase is equal to $\mathbf{k} \cdot \mathbf{b}$, with $\mathbf{b}$ the Burgers vector of the dislocation. In particular, this is a momentumdependent Berry phase resulting from adiabatic motion in real space. Hence, $f_{x \bar{y}}$ is nonzero. It can be shown explicitly [44] in the perturbative regime that strain typically leads to such a Berry phase.

\section{B. Example: 2D phase space}

We first consider the case where our real-space theory consists of a single filled band living in 1D and that a 2D CS response term in phase space with a background field describes the expected responses. We will, for simplicity, only consider Abelian physics in this example. Consider the CS action

$$
S_{\mathrm{CS}}=\frac{1}{4 \pi} \int d t d^{2} x C(\bar{x}, x) \epsilon \mathcal{A} \partial \mathcal{A} .
$$

(In Sec. IV, we show, in an explicit model, how Eq. (9), with this (quantized) coefficient, appears, but for now we simply assume that it is the relevant response theory.) Here, 
$C(\bar{x}, x)$ accounts for the filling at different points; for example, if the system occupies $x>0$, then $C(\bar{x}, x)$ will be proportional to $\Theta(x)$, with $\Theta$ the Heaviside step function, as shown in Fig. 1(a). Likewise, if the system has a Fermi momentum $k_{F}$, then $C(\bar{x}, x)$ will be proportional to $\left[\Theta\left(\bar{x}+k_{F} / B_{0}\right)-\Theta\left(\bar{x}-k_{F} / B_{0}\right)\right]$, as shown in Fig. 1(b).

Let us assume that there are no edges so that $C=1$ everywhere. Then, the responses for this action, given by $j^{\mu}=\delta S / \delta \mathcal{A}_{\mu}$, are

$$
j_{2 \mathrm{D}}^{\mu}=\frac{1}{4 \pi} \epsilon^{\mu \nu \sigma} \mathcal{F}_{\nu \sigma},
$$

where $\mathcal{F}_{\nu \sigma}=\partial_{\nu} \mathcal{A}_{\sigma}-\partial_{\sigma} \mathcal{A}_{\nu}$ is the field-strength tensor corresponding to $\mathcal{A}$. Let us consider each component, assuming for conciseness that the background field is in Landau gauge $A_{\bar{x}}=B_{0} x$.

First, we examine the real-space response $j_{2 \mathrm{D}}^{x}=\mathcal{F}_{\bar{x} t} / 2 \pi$. This current, in general, depends on $\bar{x}$, which we interpret as $k_{x} / B_{0}$; the observable current should then be given by integrating the $2 \mathrm{D}$ current with respect to $\bar{x}$, as the realspace current has contributions from all occupied momenta. The resulting $1 \mathrm{D}$ current is

$$
j_{1 \mathrm{D}}^{x}=\frac{1}{2 \pi}\left(\int d \bar{x} \partial_{\bar{x}} a_{t}-\partial_{t} \int d \bar{x} a_{\bar{x}}\right)
$$

Interpreting the $\bar{x}$-dependent part of $a_{t}$ as the dispersion, the first integral generically gives zero. The second integral is, for a gapped system, exactly the time derivative of the polarization $P_{x}=(1 / 2 \pi) \int a_{\bar{x}} \mathrm{~d} \bar{x}$, which is the expected 1D real-space current response $j_{1 \mathrm{D}}^{x}=-\partial_{t} P_{x}$. Similarly, the $k$-space response is $j_{2 \mathrm{D}}^{\bar{x}}=\mathcal{F}_{t x} / 2 \pi$. Interpreting $j_{2 \mathrm{D}}^{\bar{x}}$ as $d k / d t$, we recover the real-space semiclassical equation of motion $d k / d t=E / 2 \pi$, with $E$ the electric field.

Finally, the charge response is given by

$$
\rho_{1 \mathrm{D}}=\frac{1}{2 \pi} \int \mathrm{d} \bar{x}\left(F_{x \bar{x}}+\partial_{x} a_{\bar{x}}-\partial_{\bar{x}} a_{x}\right) .
$$

In units $B_{0}=1$, the first term simply gives the total charge in the occupied band, which can be thought of as a quasiOD response.

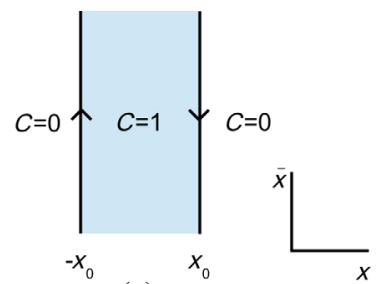

(a)

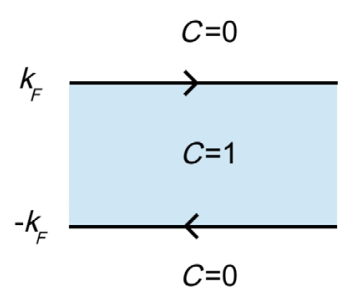

(b)
FIG. 1. Phase-space realization of (a) real-space edges and (b) Fermi points for a 1D real-space system. Arrows indicate the direction of the edge modes.
The second term of Eq. (12), in a gauge where $a_{x}=0$, becomes $\partial_{x} P_{x}$ for a gapped system. This is again intuitive; if, say, the system is strained, then the polarization and hence the charge density will change accordingly.

If we now impose a pair of edges at $\bar{x}= \pm k_{F}$, two things happen. First, the 1D system lies between a pair of momentum points $\pm k_{F}$, so the integrals in Eqs. (11) and (12) run from $-k_{F}$ to $+k_{F}$ instead of the full Brillouin zone. Consequently, the background charge becomes $\rho_{1 \mathrm{D}}^{b g}=(1 / 2 \pi) \int_{-k_{F}}^{k_{F}} F_{x \bar{x}}=k_{F} / \pi$, as expected, while the terms proportional to $f_{x \bar{x}}$ cease to have a simple interpretation as the polarization but can be nonzero nonetheless. Second, the 2D system develops a chiral anomaly at the edge, given by

$$
\partial_{t} \rho_{2 \mathrm{D}}+\partial_{x} j_{2 \mathrm{D}}^{x}=\frac{1}{2 \pi} \mathcal{F}_{t x} \partial_{\bar{x}} f(\bar{x})=\frac{E+\partial_{x} \varepsilon}{2 \pi} \partial_{\bar{x}} C(\bar{x}),
$$

where $C(\bar{x})=\Theta\left(\bar{x}+k_{F}\right)-\Theta\left(\bar{x}-k_{F}\right)$. Integrating over 1D real space under a constant electric field and translational invariance yields

$$
\partial_{t} \int d x \rho_{2 \mathrm{D}}=\frac{L}{2 \pi}\left(\delta\left(\bar{x}+k_{F}\right)-\delta\left(\bar{x}-k_{F}\right)\right) E
$$

where $L$ is the length of the system and $\delta$ is the Dirac delta function. This is precisely the chiral anomaly in the 1D system: The electric field tilts the 1D Fermi surface, effectively converting right-moving charge in the vicinity of one Fermi point into left-moving charge near the other. Thus, we have derived a property of a gapless 1D band structure from the edge anomaly of the parent 2D QH system.

Notice also that integration of (13) over momentum space leads to

$$
\partial_{t} \rho_{1 \mathrm{D}}+\partial_{x} j_{1 \mathrm{D}}^{x}=0
$$

which correctly tells us that there is no anomaly in the total charge. The precise value of $\rho_{1 \mathrm{D}}$ and $j_{1 \mathrm{D}}^{x}$ depends on system details; therefore, calculating them in our formalism would require knowledge of nonuniversal properties of the $2 \mathrm{D} Q \mathrm{QH}$ edge, such as the velocity of the chiral modes. However, we have shown here that they still have universal properties that reflect the universal properties of a higher-dimensional topological state.

A different type of anomaly occurs when the system has real-space edges and a filled band. In this case, the anomaly equation in $2 \mathrm{D}$ is

$$
\partial_{t} \rho_{2 \mathrm{D}}+\partial_{\bar{x}} j_{2 \mathrm{D}}^{\bar{x}}=-\frac{1}{2 \pi} \mathcal{F}_{t \bar{x}} \partial_{x} C(x)
$$

Integrating the above in $\bar{x}$ yields

$$
\partial_{t} \rho_{1 d}=\left(\left(\delta\left(x-x_{0}\right)-\delta\left(x+x_{0}\right)\right) \partial_{t} P(x) .\right.
$$


This is the known result [68] that charge can be adiabatically pumped from one edge of the system to the other via a time-dependent local polarization.

We thus see that the standard responses, including anomalies, that we expect in a 1D theory are retrieved from the 2D CS theory. However, detail-dependent edge responses are described in our theory only by the anomaly (or lack thereof) that they create. We expect the same procedure to generalize to higher dimensions, and we will show that the expected topological responses appear in Sec. V.

\section{EXPLICIT MODEL}

In this section, we elucidate the precise way in which a ZLL behaves as the phase space of a system in half the number of spatial dimensions. In particular, we explain why the response theory of the lower-dimensional system should be of CS form in phase space and describe the physical meaning of projecting onto the ZLL. We also answer the question of when a CS theory in $2 n D$ can be interpreted as a phase-space response theory in $n D$.

To begin, consider a massive Dirac Hamiltonian in $2 n$ dimensions coupled to the gauge field $\mathcal{A}$ defined in Sec. III:

$$
H_{2 n D}=\sum_{i=1}^{n}\left[\Gamma_{i}\left(p_{i}-\mathcal{A}_{i}\right)+\Gamma_{\bar{i}}\left(p_{\bar{i}}-\mathcal{A}_{\bar{i}}\right)\right]+\Gamma_{0} M .
$$

$\mathcal{A}$ corresponds to large constant background fields $B_{0}$ in $n$ orthogonal planes plus small fluctuations; thus, $F_{i \bar{i}}=$ $B_{0} \gg f_{i j}, f_{i \bar{j}}, f_{\bar{i} \bar{j}}$ for all $i, j \in 0, \ldots, n$. The $\Gamma$ 's are $2 n \times 2 n$ anticommuting matrices with eigenvalues \pm 1 , and they satisfy $\Gamma_{0}=\prod_{i=1}^{n} \Gamma_{i} \Gamma_{\bar{i}}$. To zeroth order in $f$, the spectrum of $H_{2 n D}$ can be easily derived by generalizing the calculation of Sec. II; it consists of Landau levels with energies $\pm \sqrt{2 k B+M^{2}}$ for positive integers $k$ together with a ZLL state that has energy $-M$ and a spinor wave function that has a $\Gamma_{0}$ eigenvalue of -1 .

We have two tasks. First, we must isolate the topological response theory of the ZLL of this system, which we expect to be a Chern-Simons theory. Second, we must relate this Hamiltonian, projected onto the ZLL of the total field, to the Hamiltonian of the real-space system.

For the first task, note that the ZLL is occupied (unoccupied) in the ground state if $M>0(M<0)$, while the occupation of all the other Landau levels is independent of the sign of $M$. This should hold for nonzero $f$ as well if $M \gg \sqrt{B_{0}}$. As a result, the response of the ZLL to $\mathcal{A}$ is given by the terms in the total response that are odd in $M$. Moreover, it is known that the two signs of $M$ correspond to a topological and a trivial insulator (which sign corresponds to which phase is determined by the regularization far away from the Dirac point). Therefore, the difference between their response theories, which by definition is the topological part of the effective action, equals the response of the ZLL. In the absence of vertex corrections, this is known to be the $2 n$-dimensional CS action with coefficient 1 to lowest order in the coupling constant $e$. In short, the response of the ZLL is precisely the CS action with coefficient 1 in appropriate dimensions under suitable well-controlled perturbative approximations. We emphasize that this statement is true even if $H_{2 n D}$ is modified at high energies to change the total ( $n$ th) Chern numbers of the occupied and unoccupied bands. The only requirement is that the Chern numbers of the $M>0$ and $M<0$ cases differ by unity; their actual values are irrelevant for determining the ZLL response.

Next, we recall that $\left[x_{i}, \bar{x}_{i}\right]=i$ in the ZLL as shown in Sec. II, so $x_{i}$ and $\bar{x}_{i}$ can be thought of as a pair of canonically conjugate position and momentum variables. Therefore, projecting $H_{2 n D}$ onto the ZLL gives an $n$-dimensional system whose response theory is guaranteed to be of CS form in phase space. In this response theory, the gauge fields in the "momentum" directions are to be reinterpreted as momentum-space Berry connections. This flow of logic is depicted in Fig. 2 (where we have renamed $H_{2 n D}$ as $H_{\text {Dirac }}^{M}$ to make the figure self-contained).

Having shown that the response of the $n$-dimensional system is given by the phase-space CS theory, we turn to our second task and show in detail how the Hamiltonian in $n$ dimensions is related to $H_{2 n D}$. For clarity, we choose $n=1$; i.e., we demonstrate this in $1 \mathrm{D}$ real space with a $U(1)$ gauge field. The procedure generalizes

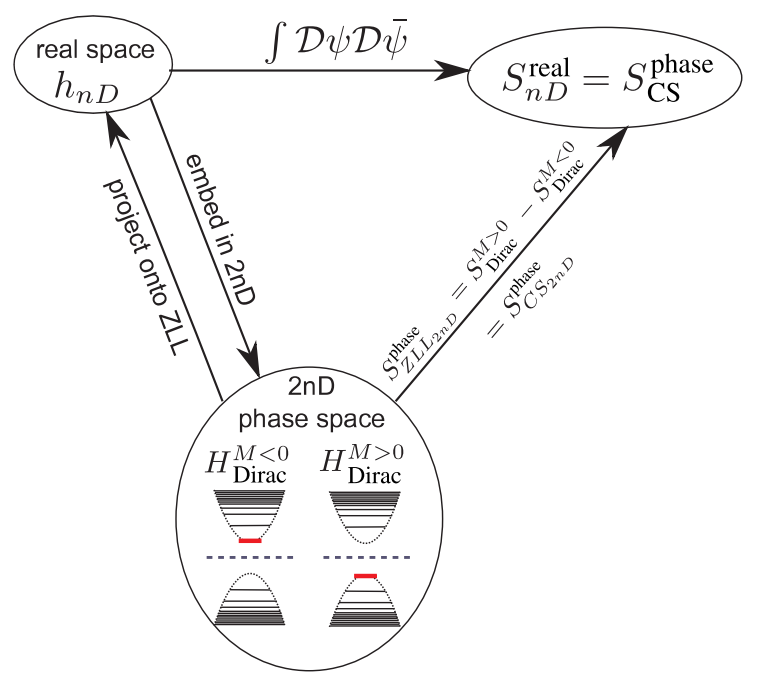

FIG. 2. Logical flow of the derivation in Sec. IV. An $n D$ Hamiltonian $h_{n D}$ can be obtained by projecting a $2 n D$ massive Dirac Hamiltonian $H_{\text {Dirac }}^{M}$ in a magnetic field onto the ZLL, denoted by the thick red bar. For a Fermi level in the Dirac mass gap, the $M>0$ and $M<0$ ground states differ only in the occupation of the ZLL, while their response theories $S_{\text {Dirac }}^{M}$ differ by the CS term in $2 n D$. Thus, the response theory of the ZLL $S_{\mathrm{ZLL}_{2 n D}}$, which is the real-space response theory $S_{n D}^{\text {real }}$ of $h_{n D}$, is the phase-space CS theory $S_{\mathrm{CS}}^{\text {phase }}$. 
straightforwardly to more dimensions and to larger gauge groups. To construct the 2D phase-space model, let $\Gamma_{x}, \Gamma_{\bar{x}}$, and $\Gamma_{0}$ be the Pauli matrices $\sigma_{x}, \sigma_{y}$, and $\sigma_{z}$. [The $\Gamma$ notation is for consistency with the higher-dimensional generalization in Eq. (18).] The appropriate 2D Hamiltonian is

$$
H_{2 \mathrm{D}}=\left(p_{x}-\mathcal{A}_{x}\right) \Gamma_{x}+\left(p_{\bar{x}}-\mathcal{A}_{\bar{x}}\right) \Gamma_{\bar{x}}+M \Gamma_{0}+\mathcal{A}_{0} .
$$

We are projecting onto the ZLL of the total field $\mathcal{A}$, so we need to make some approximations to make progress. We assume that the field fluctuations $f$ are much smaller than $B_{0}$. In other words, we identify $1 / \sqrt{B_{0}}$ with some microscopic length scale like a lattice constant for the underlying real-space system, and assume that all the gauge-field fluctuations are small over that length scale. If this is true, then we can make the gauge choice that $\partial_{\mu} a_{\nu} \ll B_{0}$ for all $\mu, \nu$. In this case, the Hamiltonian of the ZLL of $\mathcal{A}$ can be computed by considering $a$ to be a perturbation on the Hamiltonian with $\mathcal{A}=A$. We implement degenerate perturbation theory as follows.

Let us write

$$
H_{2 \mathrm{D}}=H_{0}+H^{\prime}
$$

with

$$
H_{0}=\left(p_{x}-A_{x}\right) \Gamma_{x}+\left(p_{\bar{x}}-A_{\bar{x}}\right) \Gamma_{\bar{x}}+M \Gamma_{0}-A_{0},
$$

$$
H^{\prime}=-a_{x} \Gamma_{x}-a_{\bar{x}} \Gamma_{\bar{x}}-a_{0} .
$$

Since $A$ is a constant background field of strength $B_{0}$, we know how to diagonalize $H_{0}$; let $|n, k\rangle$ be the eigenstates, where $n$ labels the LL and $k$ labels a momentum (in Landau gauge). Using $\langle\bar{x} \mid 0, k\rangle \propto e^{-B_{0}\left(\bar{x}-k / B_{0}\right)^{2} / 2}(1,0)$, where the spinor indicates that the ZLL states are polarized in the basis of $\Gamma_{0}$ eigenstates, and denoting $k_{ \pm}=k \pm q / 2$, firstorder degenerate perturbation theory gives an effective 1D Hamiltonian as

$$
\begin{aligned}
\left\langle 0, k_{-}\left|H^{\prime}\right| 0, k_{+}\right\rangle & \propto-\int d x d \bar{x} e^{i q x} a_{0}(x, \bar{x}) e^{-B_{0}\left(\bar{x}-k / B_{0}\right)^{2}} e^{-q^{2} / 4 B_{0}} \\
& \propto-a_{0}\left(-i \delta^{\prime}(q), k / B_{0}\right) \\
\Rightarrow h_{1 d}(k) & \equiv-a_{0}\left(i \partial_{k}, k / B_{0}\right) .
\end{aligned}
$$

Thus, the desired 1D Hamiltonian $h_{1 d}(x, k)$ can easily be obtained by choosing $a_{0}(x, \bar{x})=-h_{1 d}\left(x, B_{0} \bar{x}\right)$. Since the ZLL is spin polarized, the dependence on $a_{x}$ and $a_{\bar{x}}$ disappears from Eq. (23); these fields only appear at second order in perturbation theory. Degenerate perturbation theory tells us that, if $P$ is the projector onto the degenerate subspace, then the second-order correction to the energy is given by the eigenvalues of

$$
\left\langle\psi_{i}\left|H_{2 \mathrm{D}}\right| \psi_{j}\right\rangle=\left\langle 0, k_{i}\left|\left(H_{0}+H^{\prime}+\left(H^{\prime}-P H^{\prime} P\right)\left(H_{0}-E_{0}\right)^{-1}\left(H^{\prime}-P H^{\prime} P\right)\right)\right| 0, k_{j}\right\rangle \equiv\left\langle 0, k_{i}\left|H_{2 \mathrm{D}}+H^{(2)}\right| 0, k_{j}\right\rangle,
$$

where

$$
\left|\psi_{i}\right\rangle=\left|0, n_{i}\right\rangle+\sum_{n>0, l}\left|n, k_{l}\right\rangle\left\langle n, k_{l}\left|\left(H_{0}-E_{0}\right)^{-1}\left(H^{\prime}-P H P\right)\right| n=0, k_{i}\right\rangle
$$

is a basis for the perturbed ZLL wave functions up to first order in $H^{\prime}$. In particular, the unitary transformation $U$ that takes $H_{2 \mathrm{D}}$ to $H_{2 \mathrm{D}}+H^{(2)}$, to second order in $H^{\prime}$, is the one that takes $\left|0, k_{i}\right\rangle$ to a state living in the ZLL of the full Hamiltonian, to first order in $H^{\prime}$.

Therefore, if we find this unitary transformation and then perform the projection in the ZLL of the background field, we still get our desired projected Hamiltonian. We write $U=\exp (i S)$, with $S$ Hermitian, and expand $S=S_{1}+S_{2}+\ldots$ where the subscripts indicate an expansion in orders of $H^{\prime}$ (by inspection, $S$ can be chosen to have no zeroth-order term). Then, we can match, order by order, terms in $e^{i S} H_{2 \mathrm{D}} e^{-i S}$ with those in $H_{2 \mathrm{D}}+H^{(2)}$ to find the conditions

$$
\begin{gathered}
{\left[H_{0}, S_{1}\right]=0,} \\
{\left[H_{0}, S_{2}\right]=i H^{(2)} .}
\end{gathered}
$$

We do not claim to be able to demonstrate explicitly a unitary transformation that obeys the second of these conditions, as computing $H^{(2)}$ is highly nontrivial. However, we will proceed first by exhibiting an ansatz for $U$, then showing that the projection onto the ZLL of $A$ yields the correct Hamiltonian in real space, and finally giving the physical motivation for the ansatz.

Let us start in the gauge $A_{x}=-B_{0} \bar{x}, A_{\bar{x}}=A_{t}=0$. Then, let

$$
U=\left(e^{i a_{\bar{x}} p_{x} d s / B_{0}}\right)^{N} e^{-i B_{0} x \bar{x}}\left(e^{-i a_{x} p_{\bar{x}} d s / B_{0}}\right)^{N} e^{i B_{0} x \bar{x}},
$$

where $d s$ is an infinitesimal parameter and $N \rightarrow \infty$ such that $N d s=1$. This transformation is a gauge transformation, followed by a translation of $\bar{x}$ by $-a_{x}$, followed by the reverse gauge transformation, followed by a translation of $x$ by $a_{\bar{x}}$.

By inspection, $U$ commutes with $H_{0}$, so Eq. (26) is satisfied. To second order in $H^{\prime}$, we now have 


$$
U H_{2 \mathrm{D}} U^{\dagger} \approx H_{0}-a_{x}\left(x+\frac{a_{\bar{x}}}{B_{0}}, \bar{x}-\frac{a_{x}}{B_{0}}\right) \Gamma_{x}-a_{\bar{x}}\left(x+\frac{a_{\bar{x}}}{B_{0}}, \bar{x}-\frac{a_{x}}{B_{0}}\right) \Gamma_{\bar{x}}-a_{0}\left(x+\frac{a_{\bar{x}}}{B_{0}}, \bar{x}-\frac{a_{x}}{B_{0}}\right)
$$

where $a_{i}$ appearing without explicit functional dependence means $a_{i}(x, \bar{x})$. The terms that we have neglected are "double-nestings" of $a / B_{0}$; our aforementioned approximation that $a$ is slowly varying (which was a gauge choice possible when the corresponding field strengths were weak) allows us to write

$$
a_{0}\left(x+\frac{a_{\bar{x}}}{B_{0}}, \bar{x}-\frac{a_{x}\left(x+\frac{a_{\bar{x}}}{B_{0}}, \bar{x}\right)}{B_{0}}\right) \approx a_{0}\left(x+\frac{a_{\bar{x}}}{B_{0}}, \bar{x}-\frac{a_{x}}{B_{0}}\right) .
$$

Let us now perform the projection on the ZLL of the background field. As before, everything projects to zero except for the $a_{0}$ term and the mass term of $H_{0}$. The latter just projects to a constant, which we can absorb by a shift of $a_{0}$. However, we now obtain a different 1D Hamiltonian $h_{1 d}^{\prime}(x, \bar{x})=-a_{0}\left(x+a_{\bar{x}}, \bar{x}-a_{x}\right)$, which is simply $h_{1 d}(x, \bar{x})$ with minimal coupling to the gauge fields $a_{\bar{x}}$ and $a_{x}$, respectively ( $B_{0}$ is set to unity for convenience). We therefore have correctly retrieved the full 1D Hamiltonian from a projection to the ZLL, as the functional form of the projected Hamiltonian is correct if we imbue $x$ and $\bar{x}$ with the interpretations of a parameter tracking a locally periodic Hamiltonian in space and Bloch momentum, respectively.

A major question remains: Why, physically, should this choice of $U$ be the correct one? First of all, the projected Hamiltonian, if it is to describe a real system, must be gauge invariant. Hence, the gauge fields should be minimally coupled, and $U$ indeed accomplishes this goal.

A more fundamental reason, though, is the following. Consider $H_{2 \mathrm{D}}$ in some local region over which $a$ is approximately constant, and for convenience, choose a gauge in which $a_{\bar{x}}$ is zero. In this region, $a_{x}$ functions as a constant shift of the momentum $p_{x}$ which dictates, in the ZLL of $A$, the wave-function center in $\bar{x}$. Hence, we should, roughly speaking, identify the (local) eigenvalue of $p_{x}-a_{x}$ with $\bar{x}$. In the original basis, then, the variable canonically conjugate to $x$ is identified in the ZLL with $\bar{x}+a_{x}$. If we are to interpret the commutator of the projected $x$ and $\bar{x}$ operators in phase space as being the canonical commutation relation of $x$ and $p$ in real space, then we need to shift $\bar{x}$ by $-a_{x}$ in order to do so. By a similar argument in the gauge where $a_{x}=0$, we should shift $x$ by $a_{\bar{x}}$ to identify $x$ with $p_{\bar{x}}$ in the ZLL.

Having derived the real-space Hamiltonian from an ansatz for the solution to the phase-space one, we now comment on a few details.
First, notice that this derivation generalizes easily to higher dimensions, as the background field only couples $x$ to $\bar{x}, y$ to $\bar{y}$, etc. The primary difference is that in $2 n$-dimensional phase space, the $\Gamma$ matrices must be anticommuting elements of the Clifford algebra of $2 n \times 2 n$ matrices with $\operatorname{diag}\left(\Gamma_{i}\right)=0$ for $i \neq 0$.

We next comment on gauge invariance. It may appear that there is extra gauge invariance in the phase-space theory; in particular, it may seem strange that the Berry connections $a_{\bar{x}}$ can be gauge transformed into real-space gauge fields $a_{x}$ and vice versa. We claim that this is simply a reflection of the usual gauge invariance in the lowerdimensional Hamiltonian. To see this, consider a unitary operator $U=\exp [i f(x, \bar{x})]$ which implements the gauge transformation $a_{\mu} \rightarrow a_{\mu}+\partial_{\mu} f$, and let the ZLL wave functions be $|n\rangle$ for some set of labels $n$. Since $U$ is a gauge transformation in the phase-space system, it must commute with the projection operator $P$ (as $U$ must take states in a given LL to the same LL). Hence, we can project $U$ to get its action on the projected Hamiltonian; by the same argument we used for projecting the Hamiltonian, we must have $P U P=\exp [i f(x, k)]$. To understand the meaning of this operator, recall that, locally, any state can be labeled as a Bloch wave function $|k ; x\rangle$ at momentum $k$ for a local Hamiltonian at $x$. Therefore, a gauge transformation in the higher-dimensional system is equivalent to a spatially dependent $U(1)$ gauge transformation on the eigenstates $|k ; x\rangle$ of the local Hamiltonian parametrized by $x$.

Finally, after seeing the derivation, we may answer the following question: When can a CS theory in $2 n D$ be interpreted as the phase-space response theory of a system in $n D$ ? The key physical requirement in our derivation was that the total field in the $2 n D$ system could be separated into two parts: a uniform background field, which sets some length scale, and another portion which varies slowly on that length scale. When this condition holds, the CS theory may be interpreted as a phase-space theory for some lower-dimensional system.

\section{ENUMERATION OF BULK RESPONSES}

Having shown that the phase-space CS theory is the correct unified theory, we now systematically enumerate all the bulk responses of the CS theory for each possible dimensionality of phase space, and interpret them in real space. To avoid cluttering the notation, we set $B_{0}=1$.

The $2 \mathrm{D}$ responses were discussed in Sec. III B. There, we showed that the real-space current density is the rate of change of polarization, while the $k$-space current density reflects the expected relation $d k / d t \sim E$, with $E$ the electric 
TABLE II. Summary of 2D phase-space responses.

\begin{tabular}{lc}
\hline \hline Current component & Response \\
\hline Real space & Change in polarization \\
$k$ space & Electric force \\
Charge density & Band filling \\
\hline \hline
\end{tabular}

field. The charge-density response is just the band filling corrected for strain-induced changes in the lattice constant.

We summarize the 2D responses in Table II.

\section{A. 4D phase space}

The action is given by

$$
S=\frac{1}{24 \pi^{2}} \int d t d^{4} x \operatorname{tr}[C(x, \bar{x}) \epsilon \mathcal{A} \partial \mathcal{A} \partial \mathcal{A}+\cdots]
$$

where $+\cdots$ indicates the non-Abelian terms. We set $C=1$ uniformly to look at bulk responses.

Spatial components.-Without further information about the system under consideration there is no difference between the spatial directions $x$ and $y$, so we focus on the $x$ responses,

$$
j_{2 \mathrm{D}}^{x}=\frac{1}{4 \pi^{2}} \int d^{2} \bar{x} \operatorname{tr}\left[\mathcal{F}_{y \bar{y}} \mathcal{F}_{t \bar{x}}+\mathcal{F}_{\bar{x} \bar{y}} \mathcal{F}_{t y}+\mathcal{F}_{y \bar{x}} \mathcal{F}_{\bar{y} t}\right] .
$$

The first term includes the background field; setting $\mathcal{F}_{y \bar{y}}=F_{y \bar{y}}=B_{0}$ turns this into $j_{2 \mathrm{D}}^{x}=$ $(1 / 2 \pi) \int d \bar{x} \operatorname{tr}\left[\mathcal{F}_{t \bar{x}}\right]=\partial_{t} P_{x}$, with $P_{x}$ the polarization in the $x$ direction. This is the same response that appears in $1 \mathrm{D},=$ and is illustrated in Fig. 3(a). The second term is the anomalous Hall response; in the simple case where $\mathcal{F}_{t y}$ is simply an electric field, this term gives a current $j^{x}=E_{y} \int d^{2} \bar{x} \operatorname{tr}\left[\mathcal{F}_{\bar{x} \bar{y}}\right] / 4 \pi^{2}=E_{y} C_{1} / 2 \pi$, with $C_{1}$ the first Chern number of the occupied bands. This formula also

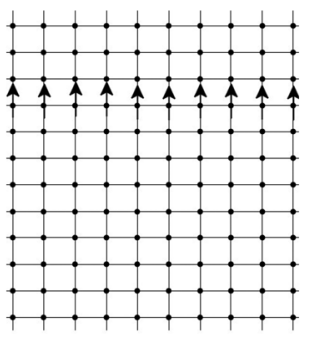

(a)

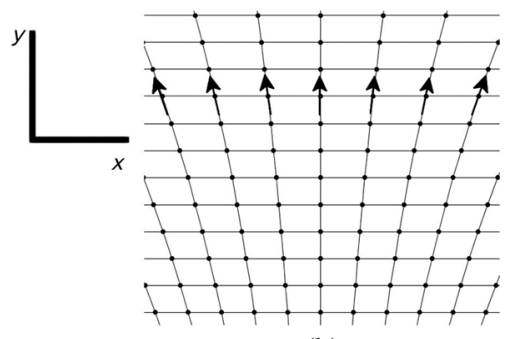

(b)
FIG. 3. Cartoon of the polarization response of an (a) unstrained square lattice and (b) sheared square lattice. In (b), $F_{y \bar{x}} \neq 0$ because motion along the $y$ lattice vector leads to translation in the unstrained $x$ direction. The directed lines are the flow of charge due to a positive $\partial_{t} P_{y}$; the polarization is measured along the lattice vector, which is deformed in (b) because of strain. Only case (b) has a nonzero current in the $x$ direction, which is due to this deformation. applies to systems with open boundaries in the $\bar{x}, \bar{y}$ directions, in which case $C_{1}$ is not quantized but still determines the intrinsic Hall conductivity of the twodimensional Fermi liquid [41-45].

The third term, illustrated in Fig. 3(b), says the following. Suppose that there is a change in time of the polarization in the $y$ direction, i.e. $\mathcal{F}_{\bar{y} t} \neq 0$, without any strain in the system. If we now add shear in the system, i.e., have $\mathcal{F}_{y \bar{x}} \neq 0$, then some of that polarization change becomes a current along the $x$ direction as defined before adding strain.

$k$ space components.-The $\bar{x}$-direction responses are

$$
j_{4 \mathrm{D}}^{\bar{x}}=\frac{1}{4 \pi^{2}} \operatorname{tr}\left[\mathcal{F}_{t x} \mathcal{F}_{y \bar{y}}+\mathcal{F}_{t y} \mathcal{F}_{\bar{y} x}+\mathcal{F}_{t \bar{y}} \mathcal{F}_{x y}\right]
$$

The first term is quasi-1D, which means that $d k_{x} / d t$ is proportional to the electric field $E_{x}$. The second term says that an electric field $E_{y}$ leads to a change in $k_{x}$ if there is shear in the system. The third term is, semiclassically, the Lorentz force-changing the polarization in the $y$ direction $\left(\mathcal{F}_{t \bar{y}} \neq 0\right)$ leads to a $1 \mathrm{D}$ current in the $y$ direction, which then feels the Lorentz force of the magnetic field $\left(\mathcal{F}_{x y} \neq 0\right)$, causing $k_{x}$ to change.

Charge component.-The charge responses are

$$
j_{2 \mathrm{D}}^{t}=\frac{1}{4 \pi^{2}} \int d^{2} \bar{x} \operatorname{tr}\left[\mathcal{F}_{x y} \mathcal{F}_{\bar{y} \bar{x}}+\mathcal{F}_{x \bar{x}} \mathcal{F}_{y \bar{y}}-\mathcal{F}_{x \bar{y}} \mathcal{F}_{y \bar{x}}\right]
$$

The first term is the Hall response for a Chern insulator. Specifically, if $F_{x y}$ is just the magnetic field, this term becomes $j^{t}=\left(B_{z} / 4 \pi^{2}\right) \int d^{2} \bar{x} F_{\bar{y} \bar{x}}=C_{1} B_{z} / 2 \pi$, where $C_{1}$ is the first Chern number.

Consider the remaining terms

$$
j_{2 \mathrm{D}}^{t}=\frac{1}{4 \pi^{2}} \int d^{2} \bar{x} \operatorname{tr}\left[\mathcal{F}_{x \bar{x}} \mathcal{F}_{y \bar{y}}-\mathcal{F}_{x \bar{y}} \mathcal{F}_{y \bar{x}}\right]
$$

In the simplest case of a single featureless, flat band, $f_{i \bar{j}} \propto \partial_{i} u_{j}$, where $\mathbf{u}$ is the displacement vector. This proportionality occurs because infinitesimal motion $d x_{i}$ in the $i$ direction leads to a translation $d x_{j}=\partial_{i} u_{j} d x_{i}$ in the $j$ direction, which is, for Bloch wave functions, the same as accumulating a $\bar{j}$-dependent phase $B_{0} \bar{j} d x_{j}$. Hence, $a_{i}=$ $\bar{j} \partial_{i} u_{j}$ with our convention of $B_{0}=1$.

In this simple case, then, Eq. (35) becomes

$$
j_{2 \mathrm{D}}^{t}=\frac{1}{4 \pi^{2}} \int d^{2} \bar{x}\left(\left(1+\partial_{x} u_{x}\right)\left(1+\partial_{y} u_{y}\right)-\partial_{x} u_{y} \partial_{y} u_{x}\right) .
$$

The expression inside the parentheses is the determinant of the deformation gradient, that is, the area of the strained unit cell in units of the original unit-cell area. Hence, the nonbackground terms are just due to the change in the area 
TABLE III. Summary of responses from 4D phase space.

\begin{tabular}{lc}
\hline \hline Current density component & Response \\
\hline Real space & Change in polarization \\
& Hall response \\
$k$ space & Change in polarization with strain \\
& Electric force \\
Charge density & Sheared response to electric field \\
& Lorentz force \\
& Hall response \\
\hline \hline
\end{tabular}

of the unit cell. Adding features to the bands will lead to corrections due to, for example, strain changing the local density of states.

We summarize the 4D responses in Table III.

\section{B. 6D phase space}

The action is given by

$$
S=\frac{1}{192 \pi^{3}} \int d t d^{6} x \operatorname{tr}[C(x, \bar{x}) \epsilon \mathcal{A} \partial \mathcal{A} \partial \mathcal{A} \partial \mathcal{A}+\cdots]
$$

with $+\cdots$ again representing the terms for a non-Abelian gauge field. For simplicity of exposition and interpretation, we assume that the momentum-space and time components of $\mathcal{A}$ are $U(N)$ and the real-space components are $U(1)$; that is, the latter couple to all the bands in the same way. This assumption is not necessary for our theory, however. We again assume that $C=1$ uniformly to look at bulk responses.

There are 15 different responses in each component. If we separate $\mathcal{F}_{x \bar{x}}$ into its background and nonbackground components, for the spatial and momentum components we get an extra 7 terms for a total of 22. For the charge component, there are 28 . We sort them, neglecting relative minus signs between the groups.

Spatial components.-Quasi-1D response (5 terms):

$j_{3 \mathrm{D}}^{x}=\frac{1}{8 \pi^{3}} \int d^{3} \bar{x} \operatorname{tr}\left[\mathcal{F}_{t \bar{x}}\left(\left(F_{y \bar{y}}+f_{y \bar{y}}\right)\left(F_{y \bar{y}}+f_{y \bar{y}}\right)-\mathcal{F}_{y \bar{z}} \mathcal{F}_{z \bar{y}}\right)\right]$.

By the same computation that was done for the charge response in $4 \mathrm{D}, \mathcal{F}_{y \bar{y}} \mathcal{F}_{z \bar{z}}-\mathcal{F}_{y \bar{z}} \mathcal{F}_{z \bar{y}}$ is the change in area perpendicular to the current. This response thus has the form of the 1D real-space response (time-varying polarization) times the change in area perpendicular to the current.

Layered Chern insulator response (2 terms):

$$
j_{3 \mathrm{D}}^{x}=\frac{1}{8 \pi^{3}} \int d^{3} \bar{x} \operatorname{tr}\left[\mathcal{F}_{t y} \mathcal{F}_{\bar{x} \bar{y}} F_{z \bar{z}}+(y \leftrightarrow z)\right],
$$

where $(y \leftrightarrow z)$ means to switch $y$ and $z$ as well as $\bar{y}$ and $\bar{z}$. This is the Hall response corresponding to thinking of the
3D system as 2D systems layered in momentum space. Note that this includes the Hall response of a Weyl semimetal (WSM) $[34,52,53,55,69]$ appearing from its monopoles of $\mathcal{F}_{\bar{x} \bar{y}}$. This can be seen by thinking of the (two-node) WSM as stacks of 2D insulators parametrized by the momentum direction along which the Weyl nodes are split; as shown in Fig. 4, each insulator lying between the nodes is a Chern insulator and thus contributes to $\mathcal{F}_{\bar{x} \bar{y}}$ (for $k_{z}$-direction Weyl node splitting). In this special case, integration yields

$$
j_{3 \mathrm{D}}^{x}=\frac{1}{4 \pi^{2}}\left(E_{y} \Delta k_{z}-E_{z} \Delta k_{y}\right),
$$

where $\Delta k_{i}$ is the splitting of the Weyl points in the $k_{i}$ direction.

Topological magnetoelectric effect (3 terms):

$$
j_{3 \mathrm{D}}^{x}=\frac{1}{8 \pi^{3}} \int d^{3} \bar{x} \operatorname{tr}\left[\left(\mathcal{F}_{\bar{x} t} \mathcal{F}_{\bar{y} \bar{z}}-\mathcal{F}_{\bar{x} \bar{y}} \mathcal{F}_{t \bar{z}}+\mathcal{F}_{\bar{x} \bar{z}} \mathcal{F}_{t \bar{y}}\right) \mathcal{F}_{y z}\right]
$$

Assuming that $\mathcal{F}_{y z}$ does not depend on momentum for simplicity, this term is an $x$-direction current proportional to $B_{x}$. Indeed, if we assume that the real-space system is gapped so that there are no monopoles of Berry curvature, simple but tedious manipulations (see the Appendix) turn Eq. (41) into

$$
\begin{aligned}
j_{3 \mathrm{D}}^{x} & =-\frac{1}{16 \pi^{3}} \partial_{t} \int d^{3} \bar{x} \epsilon^{I J K} \operatorname{tr}\left[a_{I} \partial_{J} a_{K}+\frac{2}{3} a_{I} a_{J} a_{K}\right] B_{x} \\
& \equiv-\frac{1}{2 \pi}\left(\partial_{t} P_{3}\right) B_{x}
\end{aligned}
$$

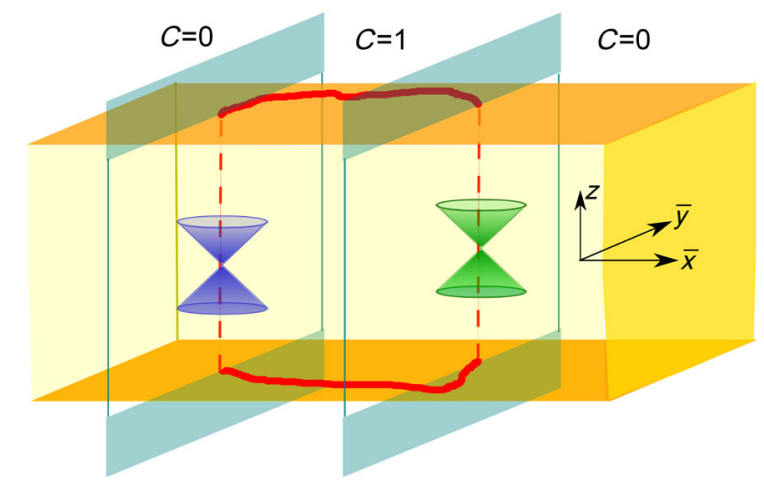

FIG. 4. Slab of WSM with Weyl nodes separated along $\bar{x}$. Each slice in momentum space with fixed $\bar{x}$ can be characterized by a Chern number $C_{1}(\bar{x})$, which changes by unity across the Weyl nodes. Thus, the region between the nodes in the above figure is a series of Chern insulators. The edge states of these Chern insulators constitute the Fermi arcs, marked as thick red lines with an irregular shape. Note that the cones are only present as a cartoon to depict the position of the Weyl nodes; the vertical direction in the figure is $z$ and should not be confused with energy. The figure is adapted from Ref. [34]. 
where $I, J, K$ run over $\bar{x}, \bar{y}, \bar{z}$ and $P_{3}$ is the threedimensional analog of charge polarization. Equation (42) is precisely the contribution of the topological magnetoelectric effect to $j^{x}$ [22].

Topological insulator (TI)-type anomalous Hall response (6 terms):

$$
\begin{aligned}
j_{3 \mathrm{D}}^{x}= & \frac{1}{8 \pi^{3}} \int d^{3} \bar{x} \operatorname{tr}\left[\mathcal{F}_{t z}\left(\mathcal{F}_{\bar{x} \bar{y}} \mathcal{F}_{y \bar{z}}-\mathcal{F}_{\bar{y} \bar{z}} \mathcal{F}_{\bar{x} y}\right)\right. \\
& \left.+\mathcal{F}_{t y} \mathcal{F}_{\bar{x} \bar{y}} f_{z \bar{z}}+(y \leftrightarrow z)\right] .
\end{aligned}
$$

If we choose a gauge such that the real-space Berry connections do not depend on momentum, then by similar logic to the topological magnetoelectric effect terms (and with similar assumptions), we can manipulate this contribution into the form

$$
j_{3 \mathrm{D}}^{x}=-\frac{1}{2 \pi}\left(E_{y} \partial_{y}-E_{z} \partial_{z}\right) P_{3} .
$$

Here, $E$ is the real-space electric field. This is the anomalous Hall effect that appears in a 3D TI [22]. It differs from the Hall effect that appears as a quasi-2D response in that it does not originate from having a nonzero total Chern number at each 2D slice of momentum space.

Sheared polarization responses (6 terms):

$$
\begin{aligned}
j_{3 \mathrm{D}}^{x}= & \frac{1}{8 \pi^{3}} \int d^{3} \bar{x} \operatorname{tr}\left[\mathcal{F}_{t \bar{y}}\left(\mathcal{F}_{\bar{x} z} \mathcal{F}_{\bar{z} y}+\mathcal{F}_{\bar{x} y}\left(F_{z \bar{z}}+f_{z \bar{z}}\right)\right)\right. \\
& +(y \leftrightarrow z)] .
\end{aligned}
$$

The first term here corresponds to a current flowing in $y$ due to a changing polarization, but that current is redirected into the $z$ and then the $x$ direction by shear. The second term is the same current in $y$ being redirected into the $x$ direction together with a change in the perpendicular area due to uniaxial strain. These are the 3D real-space analogs of the 2D real-space response illustrated in Fig. 3(b).

$k$-space components.-Quasi-1D response (5 terms):

$j^{\bar{x}}=\frac{1}{8 \pi^{3}} \operatorname{tr}\left[\mathcal{F}_{t x}\left(\left(F_{y \bar{y}}+f_{y \bar{y}}\right)\left(F_{z \bar{z}}+f_{z \bar{z}}\right)-\mathcal{F}_{y \bar{z}} \mathcal{F}_{z \bar{y}}\right)\right]$.

As in the real-space response, this is the 1D response accounting for changes in the area perpendicular to the current.

Strained electric forces (6 terms):

$j^{\bar{x}}=\frac{1}{8 \pi^{3}} \operatorname{tr}\left[\mathcal{F}_{t y}\left(\mathcal{F}_{x \bar{z}} \mathcal{F}_{\bar{y} z}+\mathcal{F}_{x \bar{y}}\left(F_{z \bar{z}}+f_{z \bar{z}}\right)\right)+(y \leftrightarrow z)\right]$.

These terms correspond to a typical electrical force in the $y$ direction, which is then redirected to the $x$ direction by shears and correcting for change in the area perpendicular to the current.

Strained polarization or Lorentz-force responses (8 terms):

$$
\begin{aligned}
j^{\bar{x}}= & \frac{1}{8 \pi^{3}} \operatorname{tr}\left[\mathcal{F}_{t \bar{y}}\left(\mathcal{F}_{z x} \mathcal{F}_{y \bar{z}}+\mathcal{F}_{x \bar{z}} \mathcal{F}_{y z}+\mathcal{F}_{x y}\left(F_{z \bar{z}}+f_{z \bar{z}}\right)\right)\right. \\
& +(y \leftrightarrow z)]
\end{aligned}
$$

The first two terms say that if the polarization changes in the $y$ direction, then either shear or the Lorentz force can change this into a current in the $z$ direction. That current can then be redirected by the Lorentz force or shear (respectively) to the $x$ direction. The third term is the same current due to polarization, leading to a current in the $x$ direction by the Lorentz force, correcting for change in the area perpendicular to the new current.

WSM-type $\mathbf{E} \cdot \mathbf{B}$ charge pumping (3 terms):

$$
j^{\bar{x}}=\frac{1}{8 \pi^{3}} \operatorname{tr}\left[\left(\mathcal{F}_{t x} \mathcal{F}_{y z}+\mathcal{F}_{t y} \mathcal{F}_{z x}+\mathcal{F}_{t z} \mathcal{F}_{x y}\right) \mathcal{F}_{\bar{y} \bar{z}}\right]
$$

Assuming the real-space field strengths are $k$ independent, this term is $\mathbf{E} \cdot \mathbf{B}$ times the Berry curvature. If we integrate over $\bar{y}$ and $\bar{z}$, we find

$$
\int d \bar{y} d \bar{z} j^{\bar{x}}=\frac{1}{4 \pi^{2}} C_{1}(\bar{x}) \mathbf{E} \cdot \mathbf{B},
$$

where $C_{1}(\bar{x})$ is the Chern number of the slice of the Brillouin zone at fixed $\bar{x}$. For the case of a WSM with Weyl points split along the $\bar{x}$ direction, $C_{1}(\bar{x})$ is nonzero between the Weyl points (see Fig. 4), so this response is a current from one Weyl point to the other. This result is exactly the chiral anomaly [34,51-53,55,69,70], which says that an $\mathbf{E} \cdot \mathbf{B}$ field in a WSM pumps charge from one Weyl point to the other.

Charge component.-All 16 terms which only contain mixed field strengths $\mathcal{F}_{i \bar{j}}$ combine to form the unitcell volume, corrected for strain. The other two types of response are as follows.

Layered Chern insulator Hall response (3 terms):

$$
j^{0}=\frac{1}{8 \pi^{3}} \int d^{3} \bar{x} \operatorname{tr}\left[\mathcal{F}_{x z} \mathcal{F}_{\bar{z} \bar{x}} F_{y \bar{y}}+\text { perm }\right]
$$

where "perm" indicates terms created by cyclically permuting $(x, y, z)$ and $(\bar{x}, \bar{y}, \bar{z})$. This is the charge density counterpart to the spatial layered Chern insulator Hall response; it analogously comes from adding the Hall response of each subsystem at fixed $k_{i}$ to a magnetic field $B_{i}$. 
TABLE IV. Summary of 6D phase-space responses.

\begin{tabular}{|c|c|}
\hline Current component & Response \\
\hline \multirow[t]{5}{*}{ Real space } & Quasi-1D responses \\
\hline & Quasi-2D (layered Chern insulator) response \\
\hline & Topological magnetoelectric effect \\
\hline & TI-like anomalous Hall response \\
\hline & Change in polarization with strain \\
\hline \multirow[t]{4}{*}{$k$ space } & Quasi-1D response \\
\hline & Electric fields with strain \\
\hline & $\begin{array}{c}\text { Change in polarization plus Lorentz } \\
\text { force with strain }\end{array}$ \\
\hline & WSM-like $\mathbf{E} \cdot \mathbf{B}$ charge pumping \\
\hline \multirow[t]{3}{*}{ Charge density } & $\begin{array}{l}\text { Density response to change } \\
\text { in unit-cell volume }\end{array}$ \\
\hline & Layered Chern insulator Hall response \\
\hline & TI-like anomalous Hall response \\
\hline
\end{tabular}

TI-type Hall response (9 terms):

$$
\begin{aligned}
j^{0}= & \frac{1}{8 \pi^{3}} \int d^{3} \bar{x} \operatorname{tr}\left[\mathcal{F}_{x z}\left(\mathcal{F}_{\bar{y} \bar{z}} \mathcal{F}_{y \bar{x}}+\mathcal{F}_{\bar{x} \bar{y}} \mathcal{F}_{y \bar{z}}+\mathcal{F}_{\bar{z} \bar{x}} f_{y \bar{y}}\right)\right. \\
& + \text { perm }] .
\end{aligned}
$$

By the same methods used to derive Eq. (42), these terms can be manipulated into the form

$$
j^{0}=\frac{1}{2 \pi} \mathbf{B} \cdot \nabla P_{3} .
$$

This is exactly the charge component of the Hall response that appears in a TI [22].

We summarize the $6 \mathrm{D}$ responses in Table IV.

\section{ANOMALIES}

In the previous section, we enumerated the phase-space bulk responses, which, as we have seen, correspond to the topological responses of filled states in real space. This includes the responses of insulators and semimetals as well as the responses of metals that involve all the occupied states, such as the anomalous Hall effect. We now wish to describe the topological features of Fermi surfaces and realspace system edges. We also approach the response of semimetals from another perspective. All these features take the form of anomalies in phase space.

Given the CS theory for a phase-space system, such as that which appears in Eq. (9) or its higher-dimensional and/ or non-Abelian generalization, suppose that $\partial_{i} C \neq 0$ for some coordinate $i$. This means that the phase-space system contains an edge; that is, the real-space system has a Fermi surface or an edge. Then, in general, the responses of the system will depend on details; for example, edge currents of quantum Hall systems depend on the nonuniversal edge mode velocity. However, there will be a universal anomaly (or lack thereof) along such edges. We see this from the anomaly resulting from the phase-space CS term in $2 n \mathrm{D}$ :

$$
\sum_{\mu \neq i} \partial_{\mu} j^{\mu}=\frac{1}{n ! 2^{2 n} \pi^{n}} \partial_{i} C \epsilon^{i \alpha_{1} \alpha_{2} \ldots \alpha_{2 n}} \operatorname{tr}\left[\mathcal{F}_{\alpha_{1} \alpha_{2}} \ldots \mathcal{F}_{\alpha_{2 n-1} \alpha_{2 n}}\right] .
$$

From here, integration over the appropriate phase-space directions determines the anomalies in the real system. We list some physically interesting effects below.

\section{A. Fermi surface anomalies}

In Sec. III B, we computed the chiral anomaly in 1D momentum space by imposing edges at $\bar{x}= \pm k_{F}$ in 2D phase space. Integrating over $x$ yielded the fact that an electric field pumps electrons in states near $+k_{F}$ into states near $-k_{F}$, or vice versa. At a down-to-earth level, this simply corresponds to tilting of the 1D Fermi surface in an electric field due to semiclassical motion of the electrons.

This idea is straightforward to generalize to higher dimensions. For instance, an open Fermi surface in 2D is obtained by confining the 4D phase-space system between $\bar{x}= \pm k_{F}$ while leaving the other three directions infinite, while a 3D spherical Fermi surface results from making the $\bar{x}, \bar{y}$, and $\bar{z}$ directions in $6 \mathrm{D}$ phase space finite under the constraint $\bar{x}^{2}+\bar{y}^{2}+\bar{z}^{2}=k_{F}^{2}$ and leaving the $x, y$, and $z$ directions unconstrained. For each phase-space geometry, the corresponding anomaly characterizes properties of the resultant Fermi surface. Importantly, if a special object such as a Dirac or a Weyl point is buried under the Fermi surface, its observable effects in local transport phenomena should emerge from the anomaly equation.

We demonstrate this first for a 3D spherical Fermi surface, which carries a Chern number, in general, and exhibits a chiral anomaly proportional to the Chern number and the electromagnetic field $\mathbf{E} \cdot \mathbf{B}$. The most well-known occurrence of this phenomenon is in Weyl semimetals. We begin with the anomaly equation in 6D phase space. In the absence of any strains and ignoring quasi-lowerdimensional terms (i.e., terms such as $F_{x \bar{x}}$ that contain the background field), it reads

$$
\begin{aligned}
& \sum_{\mu} \partial_{\mu} j^{\mu}-\partial_{\bar{r}} j^{\bar{r}} \\
& \quad=\frac{1}{8 \pi^{3}} \delta\left(\bar{r}-k_{F}\right) \mathcal{F}_{\bar{\theta} \bar{\phi}}\left(\mathcal{F}_{t x} \mathcal{F}_{y z}+\mathcal{F}_{t y} \mathcal{F}_{z x}+\mathcal{F}_{t z} \mathcal{F}_{x y}\right),
\end{aligned}
$$

where $(\bar{r}, \bar{\theta}, \bar{\phi})$ are the spherical coordinates corresponding to $(\bar{x}, \bar{y}, \bar{z})$. Integrating over the barred coordinates immediately yields the chiral anomaly in Weyl semimetals:

$$
\sum_{\mu=t, x, y, z} \partial_{\mu} j_{3 \mathrm{D}}^{\mu}=\frac{1}{4 \pi^{2}} C_{F S} \mathbf{E} \cdot \mathbf{B}
$$


where $C_{\mathrm{FS}}=\frac{1}{2 \pi} \oint \mathrm{d} \bar{\theta} \mathrm{d} \bar{\phi} \sin \bar{\theta} \mathcal{F}_{\overline{\theta \phi}} \in \mathbb{Z}$ is the Chern number of the Fermi surface which equals the total chirality of all Weyl points enclosed by it.

Unlike in 3D, in 2D systems the one-dimensional Fermi surface carries a nonquantized Berry phase instead of a Chern number. An analogous analysis, i.e., starting with the anomaly equation in 4D phase space with a $(\bar{x}, \bar{y})$ boundary that satisfies $\bar{x}^{2}+\bar{y}^{2}=k_{F}^{2}$ and integrating over $(\bar{x}, \bar{y})$, gives

$$
\sum_{\mu=t, x, y} \partial_{\mu} j_{2 \mathrm{D}}^{\mu}=\frac{1}{4 \pi^{2}} B_{z} \partial_{t} \gamma
$$

ignoring strain and quasi-lower-dimensional terms, where $\gamma=\oint_{\mathrm{FS}} \mathbf{a}_{\overline{\mathbf{r}}} \cdot \mathrm{d} \overline{\mathbf{r}}$ is the Berry phase on the Fermi surface. Equation (57) is the statement that adiabatically changing the Hall conductivity of an anomalous Hall metal in a magnetic field creates charged excitations bound to the field.

In the presence of strains, both Eqs. (56) and (57) contain more terms on their right-hand sides. We encounter these terms in the next subsection when we discuss the effects of dislocations. Before moving on, however, we wish to stress that the physical anomaly in a given dimension is independent of the topology of the Fermi surface. However, certain topologies are more convenient for studying a given physical anomaly. For instance, the chiral anomaly in Weyl metals is easier to see for a spherical Fermi surface, but it can equally well be derived for open Fermi surfaces that span one or two directions in the Brillouin zone.

\section{B. Anomalies in real space \\ 1. Real-space edge}

The simplest example of a real-space edge anomaly was derived in Sec. III B, where we imposed $x$-direction edges in 2D phase space and showed that a time-dependent polarization in 1D can be used to pump charge across the length of the chain. As a more nontrivial example, consider a real, single-band 2D Chern insulator that occupies $x>0$. Then, in 4D phase space, the anomaly equation (54) with $C(x)=\Theta(x)$ reads

$$
\begin{aligned}
\partial_{t} \rho & +\partial_{y} j^{y}+\partial_{\bar{x}} j^{\bar{x}}+\partial_{\bar{y}} j^{\bar{y}} \\
& =\frac{1}{4 \pi^{2}} \delta(x)\left(\mathcal{F}_{t y} \mathcal{F}_{\bar{x} \bar{y}}-\mathcal{F}_{t \bar{x}} \mathcal{F}_{y \bar{y}}+\mathcal{F}_{t \bar{y}} \mathcal{F}_{y \bar{x}}\right) .
\end{aligned}
$$

Integrating $\partial_{\bar{x}} j^{\bar{x}}+\partial_{\bar{y}} j^{\bar{y}}$ over momentum space gives zero since there is no boundary in those directions. Hence, integrating the previous equation over momentum space gives

$$
\partial_{t} \rho_{2 \mathrm{D}}+\partial_{y} j_{2 \mathrm{D}}^{y}=\frac{1}{4 \pi^{2}} \delta(x) \int d^{2} k \mathcal{F}_{\bar{x} \bar{y}} E_{y}=\frac{1}{2 \pi} \delta(x) C_{1} E_{y},
$$

with $C_{1}$ the first Chern number of the occupied band of the 2D Hamiltonian. We have ignored quasi-1D terms and terms containing strain. Equation (59) is recognizable as the usual anomaly for a 2D Chern insulator where an electric field parallel to the edge builds up a charge density along that edge.

\section{Dislocations}

$4 D$ phase space.-The simplest example of a dislocation is an edge dislocation in 2D real space. The key feature of the dislocation, as we discussed in Sec. III A, is that, far from the dislocation line itself, electrons accumulate a Berry's phase of $\mathbf{k} \cdot \mathbf{b}$ upon encircling the dislocation. We can thus model the dislocation by a Berry connection $\left(a_{r}, a_{\theta}\right)=(0, \mathbf{b} \cdot \mathbf{k} / 2 \pi)$, leading to a $\mathbf{k}$-independent Berry curvature $\mathcal{F}_{\theta \bar{i}}=b_{i} / 2 \pi r$. Our theory breaks down at the dislocation itself because the system changes quickly on the scale of a lattice constant. We can avoid this problem by keeping the Berry connection but surrounding the dislocation by a finite-size puncture in the system of radius $r_{0}$, i.e., choose $C=\Theta\left(r-r_{0}\right)$, with $r$ the radial coordinate in the $x y$ plane. The resulting anomaly equation reads

$$
\sum_{\mu} \partial_{\mu} j^{\mu}-\partial_{r} j^{r}=\frac{1}{8 \pi^{3}}\left(\mathcal{F}_{t \bar{x}} b_{y}-\mathcal{F}_{t \bar{y}} b_{x}\right)
$$

plus quasi-1D terms on the right-hand side, which we ignore. Integrating over $\bar{x}, \bar{y}$ and $\theta$ gives the charge radiating from the core of an edge dislocation in the presence of a time-dependent polarization:

$$
\partial_{t} \rho_{2 \mathrm{D}}=\hat{\mathbf{z}} \cdot\left(\mathbf{b} \times \partial_{t} \mathbf{P}\right) .
$$

This result is shown in Fig. 5, which makes the physical picture of the anomaly clear in the limit of weakly coupled

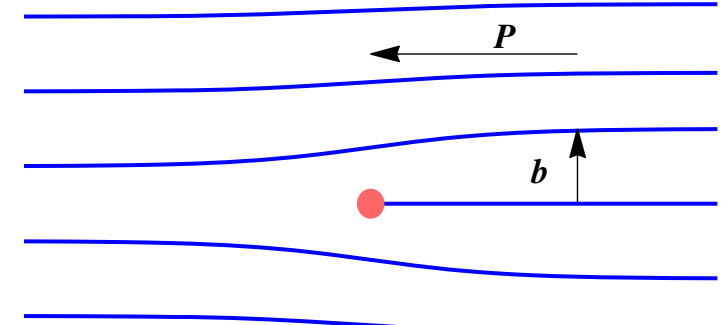

FIG. 5. An edge dislocation in 2D with the Burgers vector $\mathbf{b}$. In the presence of a polarization $\mathbf{P} \perp \mathbf{b}$, charge gets accumulated at the core of the dislocation, shown by the red dot. 
chains perpendicular to $\mathbf{b}$; the core of the dislocation is the end of such a chain, so polarizing that chain adds charge to its end. The nontrivial result is that the extra charge remains bound to the dislocation core and does not leak into other chains even when they are strongly coupled.

$6 D$ phase space.-A similar analysis for a dislocation in $3 \mathrm{D}$ real space running along $\hat{\mathbf{z}}$ and with the Burgers vector b gives

$$
\begin{aligned}
\partial_{t} \rho+ & \frac{1}{r} \partial_{\theta} j^{\theta}+\partial_{z} j^{z} \\
= & \frac{\delta\left(r-r_{0}\right)}{8 \pi^{3}} \int d^{3} \bar{x}\left(\mathcal{F}_{\bar{x} \bar{y}}\left(r \mathcal{F}_{\theta \bar{z}}\right)+\mathcal{F}_{\bar{y} \bar{z}}\left(r \mathcal{F}_{\theta \bar{x}}\right)\right. \\
& \left.+\mathcal{F}_{\bar{z} \bar{x}}\left(r \mathcal{F}_{\theta \bar{y}}\right)\right) \mathcal{F}_{t z} \\
= & -\frac{\delta\left(r-r_{0}\right)}{8 \pi^{3}} E_{z} \int d^{3} \bar{x} \Omega \cdot \mathbf{b},
\end{aligned}
$$

where $\Omega_{i}=\frac{1}{2} \epsilon_{i j k} \mathcal{F}_{\bar{j} \bar{k}}$ is the Berry curvature of the bands in the plane perpendicular to $i$.

To understand Eq. (62), let us first consider a layered Chern insulator, that is, a system composed of layers of Chern insulators stacked along a certain direction. The integral in Eq. (62) then gives the Chern number of the layers in each direction, so

$$
\partial_{t} \rho+\frac{1}{r} \partial_{\theta} j^{\theta}+\partial_{z} j^{z}=-\frac{\delta\left(r-r_{0}\right)}{\pi} E_{z} \mathbf{C} \cdot \mathbf{b},
$$

where $C_{i}=\left(1 / 8 \pi^{2}\right) \int \mathrm{d}^{3} \bar{x} \Omega_{i}$. Now, add a dislocation running along $\hat{\mathbf{z}}$ with the Burgers vector $\mathbf{b}$. Edge dislocations are defined by $\mathbf{b} \perp \hat{\mathbf{z}}$, whereas screw dislocations have $\mathbf{b} \| \hat{\mathbf{z}}$. The two scenarios are shown in Fig. 6. Equation (63) shows that in either case, there exists a chiral mode along the dislocation that participates in an anomaly in response to $E_{z}$. We can understand this as follows.
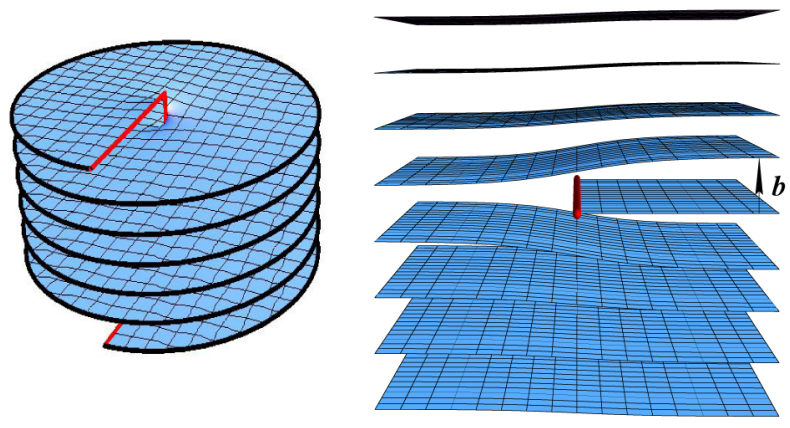

FIG. 6. Screw (left diagram) and edge (right diagram) dislocations in 3D. Dislocations in an axion insulator harbor chiral modes, denoted by red lines in both figures. In the screw dislocation, thick black lines represent the standard chiral edge mode. The screw dislocation geometry with Weyl nodes split along the screw axis was used for the numerical results presented in Fig. 7.
An edge dislocation can be thought of as a semi-infinite sheet perpendicular to $\mathbf{b}$ and unbounded along $\hat{\mathbf{z}}$ inserted into the 3D lattice. If the sheet has a Chern number, we expect it to have a chiral mode along $\hat{\mathbf{z}}$. For weakly coupled sheets, this is precisely the chiral mode along the edge dislocation. For a screw dislocation, the existence of a chiral dislocation mode follows from an argument adapted from one that predicts helical dislocation modes in weak topological insulators $[71,72]$. Suppose that our system is of finite size in the $z$ direction. Then, on each surface, there is a semi-infinite edge emerging from the dislocation. However, this edge must carry a chiral mode since the surface layer is a Chern insulator. By charge conservation, this chiral mode cannot terminate at the dislocation, so the chiral mode must proceed along the dislocation to the other surface. Moreover, in each case, the chiral mode is expected to survive for strongly coupled layers as well, where the system is better thought of as stacked sheets in momentum space and is typically termed an axion insulator. This robustness occurs because layered Chern insulators and axion insulators are actually the same phase-there is no phase transition as the interlayer coupling is strengthened-so their topological defects such as dislocations have qualitatively similar behavior. Indeed, the presence of a chiral mode was shown explicitly for an axion insulator created from a charge-density wave instability of a WSM in Ref. [73].

Spectral flow due to dislocations in Weyl semimetals.Having seen examples of anomalies being the universal feature of gapless systems, we use our theory's anomaly machinery to derive a new result: prediction of an anomaly at dislocation lines in a WSM. This result is closely related to the case of a layered Chern insulator (axion insulator) just discussed.

Consider a WSM with two nodes split by $\mathbf{K}$ (and thus having broken time-reversal symmetry) with a dislocation along $z$. In contrast to the layered Chern insulators or axion insulators, WSMs have a gapless bulk and thus cannot support localized modes the same way that the former do. However, our theory allows us to confirm that there is indeed an anomaly at the dislocation in a WSM. The anomaly calculation is identical to the axion insulator case, except that $(1 / 4 \pi) \int \mathrm{d}^{3} x \Omega_{i}=K_{i}$ instead of $2 \pi$. The result is that the anomaly is $\delta\left(r-r_{0}\right) E_{z} \mathbf{K} \cdot \mathbf{b} / 2 \pi$, which reflects the fact that chiral modes appear only in the region of momentum space between the Weyl nodes, where the Chern number of the layers is \pm 1 . From now on, we assume $\mathbf{K}=K \hat{\mathbf{z}}$ for concreteness.

The physical interpretation of this anomaly is more subtle for the WSM than the axion insulator. In the latter case, because of the bulk gap, the anomaly means that there is a chiral zero mode on the dislocation. In the WSM case, there is no bulk gap. Furthermore, if the region carrying a nonzero Chern number is near $k_{z}=0$, then that region sees only small perturbations from the dislocation because the 
dislocation acts like a flux proportional to $k_{z}$. Hence, we should not necessarily expect a zero mode on the dislocation. On the other hand, if this region is located near $k_{z}=\pi$, then there may be such a zero mode. In general, however, the existence of a localized zero mode is not guaranteed.

Since the anomaly need not imply a localized zero mode, we numerically solved a simple $\mathbf{k} \cdot \mathbf{p}$ model for a WSM in the presence of a dislocation in order to directly verify the anomaly. The Hamiltonian we used is

$$
\begin{aligned}
H= & {\left[M_{0}+M_{1} k_{z}^{2}+M_{2}\left(k_{x}^{2}+k_{y}^{2}\right)\right] \Gamma_{5}+L_{1} k_{z} \Gamma_{4} } \\
& +L_{2}\left(k_{y} \Gamma_{1}-k_{x} \Gamma_{2}\right)+U_{0} \Gamma_{12} .
\end{aligned}
$$

Here, the anticommuting $\Gamma$ matrices are defined by $\Gamma_{1,2,3}=\sigma_{x, y, z} \tau_{x}, \Gamma_{4}=\tau_{y}, \Gamma_{5}=\tau_{z}$, and $\Gamma_{i j}=\left[\Gamma_{i}, \Gamma_{j}\right] / 2 i$, where $\sigma$ is a spin index and $\tau$ is an orbital index. This model leads to Weyl points at $\mathbf{k}= \pm\left|U_{0}\right| / L_{1} \hat{\mathbf{z}}$ when the quadratic term is neglected. It has been previously investigated in a radial geometry [55] with no dislocation. The only effect of a screw dislocation at $r=0$ with the Burgers vector $b \hat{\mathbf{z}}$ is that the dependence of the components of the wave function on the in-plane angle $\theta$ changes from $e^{i n \theta}$ to $e^{i\left(l+b k_{z} / 2 \pi\right) \theta}$, where the half-integer $l$ is the eigenvalue of $L_{z}$ in the absence of the dislocation.

We solved the discretized version of this model at fixed angular momentum for a cylinder of size $R=120$ sites at fixed angular momentum $l=1 / 2+k_{z} / 2 \pi$. For comparison, we show the band structure in the WSM phase with no dislocation in Fig. 7(a). The mode localized near $r=0$ (in blue) is always at higher energy than the Fermi arc, and it is not topological. Adding the dislocation, we see in Fig. 7(b) that now the $r=0$ mode changes from unoccupied to occupied after crossing the Weyl points; an electron has been pumped from the Fermi arc (in red) to the dislocation. This is the anomaly that we discussed above, even though there is no zero-energy mode localized on the dislocation. This system can smoothly evolve, by bringing the Weyl points together and annihilating them, into the axion insulator in Fig. 7(c). That state has a single chiral mode localized on the dislocation which crosses the band gap without mixing with the outer edge mode, as expected. In Fig. 7(d), we have a WSM with a topologically nontrivial region centered about $k_{z}=\pi$; here, there is a zero mode localized on the dislocation, and the charge pumping is more obvious than in Fig. 7(b).

The result of charge pumping due to disclinations has been previously predicted [63]. However, our picture is different from the one considered there. The claim in Ref. [63] is that a chiral magnetic field, which in our case is created by the dislocation, causes a net spontaneous current to flow. As can be seen from our picture, this is not true; an electric field is necessary to have an anomaly and thus a net current. Fundamentally, the total current must vanish in the absence of an electric field. If the current did

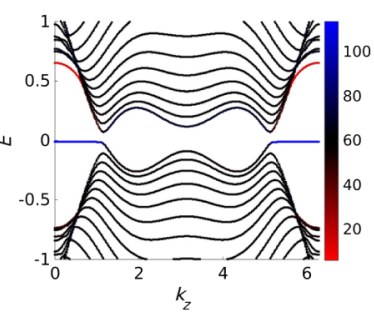

(a)

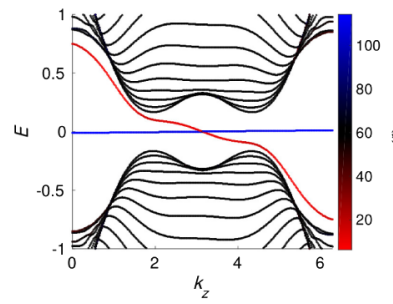

(c) (b)

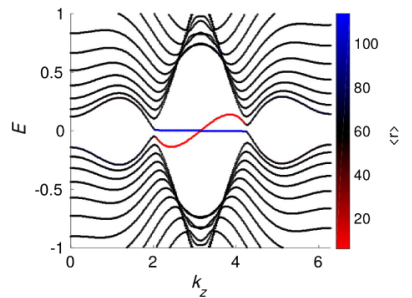

(d)

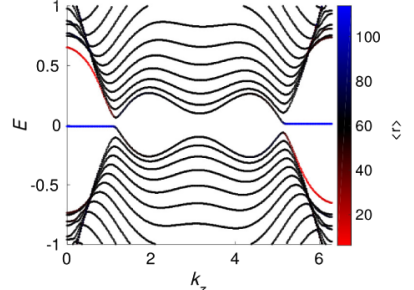

FIG. 7. Band structure of the lattice regularized version of Eq. (64) in a cylindrical geometry. Color corresponds to $\langle r\rangle$ with the dislocation at $r=0$; red indicates localization on the dislocation, and blue is localization on the outer boundary. Parameters are $M_{0}=0, M_{1}=0.342 \mathrm{eV} \AA^{2}, M_{2}=18.25 \mathrm{eV} \AA^{2}$, $L_{1}=1.33 \mathrm{eV} \AA, L_{2}=2.82 \mathrm{eV} \AA, R=120$ radial sites, and $l=$ $1 / 2$ angular momentum unless otherwise stated. Note that because of the dislocation, the system is not periodic in $k_{z}$ at fixed angular momentum. (a) WSM phase $\left(U_{0}=1.3 \mathrm{eV}\right)$, no dislocation. (b) Same as (a), but with dislocation. (c) Axion insulator phase $\left(U_{0}=1.7 \mathrm{eV}\right)$ with dislocation. (d) WSM phase $\left(U_{0}=-1.3 \mathrm{eV}, \quad M_{1}=-0.342 \mathrm{eV} \AA, \quad M_{0}=1.4 \mathrm{eV}\right) \quad$ with topologically nontrivial $\mathrm{BZ}$ slices centered at $k_{z}=\pi$ and a dislocation.

not vanish, adding an electric field parallel to the current would cause dissipation and lower the system energy, but this is impossible for a system already in its ground state. The difference in Ref. [63] stems from neglecting momentum-space regions away from the Weyl nodes and the real-space boundary in determining the total current. Thus, while the general expression for the current density derived by Ref. [63] is correct, the total current vanishes when these contributions are included. For the case that we show in Fig. 7(b), the net current due to the dislocation is canceled by the current along the Fermi arcs. In the case of Fig. 7(d), the dislocation mode near one Weyl point connects directly to the mode on the other side through a zero mode which cancels the net current.

To summarize, dislocations in a WSM indeed cause pumping of charge to (or from) the dislocation line when an electric field is applied along the dislocation. Such a charge pumping is smoothly connected to that which occurs in the axion insulator, but it may or may not, depending on details, result in a zero mode localized on the dislocation. Although we presented numerics for a screw dislocation that runs along the same direction as the Weyl node 
splitting, Eq. (62), and hence the qualitative result, is valid for edge dislocations as well as for other directions of the Weyl node splitting.

\section{DISCUSSION AND CONCLUSIONS}

We have shown that the responses and anomalies of a gapped or gapless system living in $n$ spatial dimensions can be described by a single response theory of a gapped system living in $2 n$ spatial dimensions. Conceptually, this is because adding magnetic fields in the $2 n$-dimensional system and projecting onto the zeroth Landau level allows us to interpret that system as living in phase space. We have used this theory to reproduce well-understood responses and anomalies in systems with noninteracting electrons and Abelian real-space gauge fields, as well as to demonstrate the existence of spectral flow due to dislocations in Weyl semimetals.

There are several interesting fundamental questions about our theory which are at present open. It would be interesting to see how our theory connects to the use of phase space in statistical mechanics; how might the Landau-Boltzmann transport equation, which describes transport in Fermi liquids via Wigner functions, or Liouville's theorem, which describes the time evolution of general classical systems in phase space via a density matrix, arise in our context? Both the Wigner function and the phase-space density matrix treat real and momentum space on an equal footing; thus, our theory holds promise in capturing these phenomena.

In addition to these fundamental questions, we envision a number of extensions of our theory to more complicated systems. In particular, the responses that we have explicitly discussed have so far been only those of noninteracting systems, which only feel a $U(1)$ real-space gauge field, though the $k$-space Berry connection has been allowed to be non-Abelian. The latter constraint is not an inherent limitation of the theory; perhaps there are interesting responses to a larger real-space gauge group. $S U(2)$ groups in $4 \mathrm{D}$ and 3D have been studied and shown to give topological insulator- and WSM-like responses, respectively [74]. It is thus conceivable that general gauge groups can lead to other topological responses, possibly of phases with emergent fermions such as partons [75] or composite fermions [76].

As for interactions, it is not immediately clear if there are sensible real-space systems that are well described by a phase-space theory with only local interactions. However, if there are such real-space systems, then working in phase space could be very useful because, for example, in the absence of a magnetic field, mean-field theory is more accurate because of the higher dimensionality. This advantage may be mitigated by the fact that our construction requires gauge fields, however. Alternatively, it is possible that there is a simple way to directly incorporate the interactions of the real-space system into the phase-space theory.
Another interesting question is if there is an extension of our theory that describes nodal superconductors. Our theory as written requires $U(1)$ charge conservation; perhaps there is some way to incorporate spontaneous breaking of this symmetry. Finally, it could also be interesting to explicitly incorporate other symmetries of the lower-dimensional system; this could allow a better understanding of gapless symmetry-protected phases like Dirac semimetals.

\section{ACKNOWLEDGMENTS}

D. B. is supported by the National Science Foundation under Grant No. DGE-114747. P. H. is supported by the David and Lucile Packard Foundation and the U.S. DOE, Office of Basic Energy Sciences, Contract No. DEAC0276SF00515. S. C.Z. is supported by the National Science Foundation under Grant No. DMR-1305677. X. L. Q. is supported by the National Science Foundation through Grant No. DMR-1151786.

\section{APPENDIX: TOPOLOGICAL MAGNETOELECTRIC EFFECT}

Here, we derive explicitly the topological magnetoelectric effect from our response theory. The topological magnetoelectric effect is only quantized in gapped systems, so we assume the system is gapped.

The relevant terms are in Eq. (41), which we rewrite here as

$$
j_{3 \mathrm{D}}^{x}=\frac{-1}{16 \pi^{3}} \int d^{3} \bar{x} \operatorname{tr}\left[\epsilon^{I J K} \mathcal{F}_{t I} \mathcal{F}_{J K} \mathcal{F}_{y z}\right],
$$

where $I, J, K$ run over $\bar{x}, \bar{y}, \bar{z}$. We assume that $\mathcal{F}_{y z}$ is the real-space magnetic field $B_{x}$, so we can pull it out. For simplicity, we choose a gauge such that $A_{\bar{i}}=0$ for $i=x, y, z$.

Expanding Eq. (A1) and manipulating some indices yields

$$
\begin{aligned}
j_{3 \mathrm{D}}^{x}= & -\frac{B_{x}}{8 \pi^{3}} \int d^{3} \bar{x} \epsilon^{I J K} \operatorname{tr}\left[\left(\partial_{t} a_{I}-\partial_{I} a_{t}\right.\right. \\
& \left.\left.+\left[a_{t}, a_{I}\right]\right)\left(\partial_{J} a_{K}+a_{J} a_{K}\right)\right] .
\end{aligned}
$$

We first show that several sets of terms in this expansion are zero. First, notice that

$$
\begin{aligned}
\int d^{3} \bar{x} \epsilon^{I J K} \partial_{I} a_{t} \partial_{J} a_{K} & =-\int d^{3} \bar{x} \epsilon^{I J K} \partial_{I} \partial_{J} a_{t} a_{K} \\
& =\int d^{3} \bar{x} \epsilon^{I J K} \partial_{J} a_{t} \partial_{I} a_{K} \\
& =-\int d^{3} \bar{x} \epsilon^{I J K} \partial_{I} a_{t} \partial_{J} a_{K},
\end{aligned}
$$


where we integrated by parts twice and then switched the indices $I$ and $J$. Hence,

$$
\int d^{3} \bar{x} \epsilon^{I J K} \partial_{I} a_{t} \partial_{J} a_{K}=0
$$

Next, consider the terms

$$
\begin{aligned}
\int d^{3} \bar{x} \epsilon^{I J K} \operatorname{tr}\left[\left[a_{t}, a_{I}\right] \partial_{J} a_{K}-\partial_{I} a_{t} a_{J} a_{K}\right] & =\int d^{3} \bar{x} \epsilon^{I J K} \operatorname{tr}\left[-\partial_{J}\left(a_{t} a_{I}-a_{I} a_{t}\right) a_{K}-\partial_{I} a_{t} a_{J} a_{K}\right] \\
& =\int d^{3} \bar{x} \epsilon^{I J K} \operatorname{tr}\left[\partial_{I}\left(a_{t} a_{J}-a_{J} a_{t}\right) a_{K}-\partial_{I} a_{t} a_{J} a_{K}\right] \\
& =\int d^{3} \bar{x} \epsilon^{I J K} \operatorname{tr}\left[\left(a_{t} \partial_{I} a_{J}-\partial_{I} a_{J} a_{t}-a_{J} \partial_{I} a_{t}\right) a_{K}\right] \\
& =-\int d^{3} \bar{x} \epsilon^{I J K} \operatorname{tr}\left[\left[a_{t}, a_{I}\right] \partial_{J} a_{K}-\partial_{I} a_{t} a_{J} a_{K}\right] .
\end{aligned}
$$

We have integrated by parts, manipulated indices, and used the cyclic property of the trace. Hence, the left-hand side here is also zero.

Finally, trivial manipulations show that $\int d^{3} \bar{x} \epsilon^{I J K} \operatorname{tr}\left[\left[a_{t}, a_{I}\right] a_{J} a_{K}\right]=0$ as well.

The remaining terms in the expansion are those that do not involve $a_{t}$ :

$$
\begin{aligned}
j_{3 \mathrm{D}}^{x} & =\frac{-B_{x}}{8 \pi^{3}} \int d^{3} \bar{x} \epsilon^{I J K} \operatorname{tr}\left[\partial_{t} a_{I}\left(\partial_{J} a_{K}+a_{J} a_{K}\right)\right] \\
& =\frac{-B_{x}}{8 \pi^{3}} \int d^{3} \bar{x} \epsilon^{I J K} \operatorname{tr}\left[\partial_{t}\left(a_{I} \partial_{J} a_{K}+a_{I} a_{J} a_{K}\right)-a_{I}\left(\partial_{t} \partial_{J} a_{K}+2 \partial_{t} a_{J} a_{K}\right)\right] \\
& \left.=\frac{-B_{x}}{8 \pi^{3}} \int d^{3} \bar{x} \epsilon^{I J K} \operatorname{tr}\left[\partial_{t}\left(a_{I} \partial_{J} a_{K}+a_{I} a_{J} a_{K}\right)+\partial_{J} a_{I} \partial_{t} a_{K}-2 \partial_{t} a_{I} a_{J} a_{K}\right)\right] \\
& =\frac{-B_{x}}{8 \pi^{3}} \int d^{3} \bar{x} \epsilon^{I J K} \operatorname{tr}\left[\partial_{t}\left(a_{I} \partial_{J} a_{K}+a_{I} a_{J} a_{K}\right)-\partial_{t} a_{I} \partial_{J} a_{K}-\partial_{t} a_{I} a_{J} a_{K}-\frac{1}{3} \partial_{t}\left(a_{I} a_{J} a_{K}\right)\right] \\
& =\frac{-B_{x}}{8 \pi^{3}} \int d^{3} \bar{x} \epsilon^{I J K} \operatorname{tr}\left[\partial_{t}\left(a_{I} \partial_{J} a_{K}+\frac{2}{3} a_{I} a_{J} a_{K}\right)\right]-j_{3 \mathrm{D}}^{x} .
\end{aligned}
$$

This immediately gives the desired relation (42).

[1] X.-L. Qi and S.-C. Zhang, The Quantum Spin Hall Effect and Topological Insulators, Phys. Today 63, 33 (2010).

[2] M.Z. Hasan and C. L. Kane, Colloquium: Topological Insulators, Rev. Mod. Phys. 82, 3045 (2010).

[3] X.-L. Qi and S.-C. Zhang, Topological Insulators and Superconductors, Rev. Mod. Phys. 83, 1057 (2011).

[4] M. Z. Hasan and J.E. Moore, Three-Dimensional Topological Insulators, Annu. Rev. Condens. Matter Phys. 2, 55 (2011).

[5] F. D. M. Haldane, Model for a Quantum Hall Effect without Landau Levels: Condensed-Matter Realization of the "Parity Anomaly", Phys. Rev. Lett. 61, 2015 (1988).
[6] D. J. Thouless, M. Kohmoto, M. P. Nightingale, and M. den Nijs, Quantized Hall Conductance in a Two-Dimensional Periodic Potential, Phys. Rev. Lett. 49, 405 (1982).

[7] C. L. Kane and E. J. Mele, Quantum Spin Hall Effect in Graphene, Phys. Rev. Lett. 95, 226801 (2005).

[8] C. L. Kane and E. J. Mele, $\mathbb{Z}_{2}$ Topological Order and the Quantum Spin Hall Effect, Phys. Rev. Lett. 95, 146802 (2005).

[9] R Roy, Topological Phases and the Quantum Spin Hall Effect in Three Dimensions, Phys. Rev. B 79, 195322 (2009).

[10] L. Fu, C. L. Kane, and E. J. Mele, Topological Insulators in Three Dimensions, Phys. Rev. Lett. 98, 106803 (2007). 
[11] B. A. Bernevig and S.-C. Zhang, Quantum Spin Hall Effect, Phys. Rev. Lett. 96, 106802 (2006).

[12] B. A. Bernevig, T. L. Hughes, and S.-C. Zhang, Quantum Spin Hall Effect and Topological Phase Transition in HgTe Quantum Wells, Science 314, 1757 (2006).

[13] R. Roy, $Z_{2}$ Classification of Quantum Spin Hall Systems: An Approach Using Time-Reversal Invariance, Phys. Rev. B 79, 195321 (2009).

[14] N. Read and D. Green, Paired States of Fermions in Two Dimensions with Breaking of Parity and Time-Reversal Symmetries and the Fractional Quantum Hall Effect, Phys. Rev. B 61, 10267 (2000).

[15] C. Kallin and A. J. Berlinsky, Is $\mathrm{Sr}_{2} \mathrm{RuO}_{4}$ a Chiral p-Wave Superconductor?, J. Phys. Condens. Matter 21, 164210 (2009).

[16] D. D. Osheroff, W. J. Gully, R. C. Richardson, and D. M. Lee, New Magnetic Phenomena in Liquid $\mathrm{He}^{3}$ below $3 \mathrm{mK}$, Phys. Rev. Lett. 29, 920 (1972).

[17] A. J. Leggett, Interpretation of Recent Results on $\mathrm{He}^{3}$ below 3 mK: A New Liquid Phase?, Phys. Rev. Lett. 29, 1227 (1972).

[18] A. Kitaev, Periodic Table for Topological Insulators and Superconductors, L. D. Landau Memorial Conference "Advances in Theoretical Physics" (AIP, New York, 2009), pp. 22-30.

[19] S. Ryu, A. P. Schnyder, A. Furusaki, and A. W. W. Ludwig, Topological Insulators and Superconductors: Tenfold Way and Dimensional Hierarchy, New J. Phys. 12, 065010 (2010).

[20] A. P. Schnyder, S. Ryu, A. Furusaki, and A. W. W. Ludwig, Classification of Topological Insulators and Superconductors in Three Spatial Dimensions, Phys. Rev. B 78, 195125 (2008).

[21] A. Altland and M. R. Zirnbauer, Nonstandard Symmetry Classes in Mesoscopic Normal-Superconducting Hybrid Structures, Phys. Rev. B 55, 1142 (1997).

[22] X.-L. Qi, T. L. Hughes, and S.-C. Zhang, Topological Field Theory of Time-Reversal Invariant Insulators, Phys. Rev. B 78, 195424 (2008).

[23] A. M. Essin, J. E. Moore, and D. Vanderbilt, Magnetoelectric Polarizability and Axion Electrodynamics in Crystalline Insulators, Phys. Rev. Lett. 102, 146805 (2009).

[24] Z. Wang, X.-L. Qi, and S.-C. Zhang, Topological Field Theory and Thermal Responses of Interacting Topological Superconductors, Phys. Rev. B 84, 014527 (2011).

[25] S. Ryu, J. E. Moore, and A. W. W. Ludwig, Electromagnetic and Gravitational Responses and Anomalies in Topological Insulators and Superconductors, Phys. Rev. B 85, 045104 (2012).

[26] X.-L. Qi, E. Witten, and S.-C. Zhang, Axion Topological Field Theory of Topological Superconductors, Phys. Rev. B 87, 134519 (2013).

[27] C. Wang, A. C. Potter, and T. Senthil, Classification of Interacting Electronic Topological Insulators in Three Dimensions, Science 343, 629 (2014).

[28] X. Wan, A. M. Turner, A. Vishwanath, and S. Y. Savrasov, Topological Semimetal and Fermi-Arc Surface States in the Electronic Structure of Pyrochlore Iridates, Phys. Rev. B 83, 205101 (2011).
[29] A. A. Burkov and L. Balents, Weyl Semimetal in a Topological Insulator Multilayer, Phys. Rev. Lett. 107, 127205 (2011).

[30] A. A. Burkov, M. D. Hook, and L. Balents, Topological Nodal Semimetals, Phys. Rev. B 84, 235126 (2011).

[31] A. M. Turner and A. Vishwanath, Beyond Band Insulators: Topology of Semi-metals and Interacting Phases, arXiv:1301.0330.

[32] O. Vafek and A. Vishwanath, Dirac Fermions in SolidsFrom High $T_{c}$ Cuprates and Graphene to Topological Insulators and Weyl Semimetals, Annu. Rev. Condens. Matter Phys. 5, 83 (2014).

[33] W. Witczak-Krempa and Y.-B. Kim, Topological and Magnetic Phases of Interacting Electrons in the Pyrochlore Iridates, Phys. Rev. B 85, 045124 (2012).

[34] P. Hosur and X. Qi, Recent Developments in Transport Phenomena in Weyl Semimetals, Topological Insulators/ Isolants Topologiques, C.R. Phys. 14, 857 (2013).

[35] P. Hosur, Friedel Oscillations Due to Fermi Arcs in Weyl Semimetals, Phys. Rev. B 86, 195102 (2012).

[36] A. C. Potter, I. Kimchi, and A. Vishwanath, Quantum Oscillations from Surface Fermi-Arcs in Weyl and Dirac Semi-Metals, Nat. Comm. 5, 5161 (2014).

[37] F. D. M. Haldane, Attachment of Surface "Fermi Arcs" to the Bulk Fermi Surface: "Fermi-Level Plumbing" in Topological Metals, arXiv:1401.0529.

[38] T. T. Heikkila, N. B. Kopnin, and G. E. Volovik, Flat Bands in Topological Media, JETP Lett. 94, 233 (2011).

[39] J. C. Y. Teo and C. L. Kane, Topological Defects and Gapless Modes in Insulators and Superconductors, Phys. Rev. B 82, 115120 (2010).

[40] S. Matsuura, P.-Y. Chang, A. P. Schnyder, and S. Ryu, Protected Boundary States in Gapless Topological Phases, New J. Phys. 15, 065001 (2013).

[41] R. Karplus and J. M. Luttinger, Hall Effect in Ferromagnetics, Phys. Rev. 95, 1154 (1954).

[42] F. D. M. Haldane, Berry Curvature on the Fermi Surface: Anomalous Hall Effect as a Topological Fermi-Liquid Property, Phys. Rev. Lett. 93, 206602 (2004).

[43] T. Jungwirth, Q. Niu, and A. H. MacDonald, Anomalous Hall Effect in Ferromagnetic Semiconductors, Phys. Rev. Lett. 88, 207208 (2002).

[44] D. Xiao, M.-C. Chang, and Q. Niu, Berry Phase Effects on Electronic Properties, Rev. Mod. Phys. 82, 1959 (2010).

[45] N. Nagaosa, J. Sinova, S. Onoda, A. H. MacDonald, and N. P. Ong, Anomalous Hall Effect, Rev. Mod. Phys. 82, 1539 (2010).

[46] M. Barkeshli and X.-L. Qi, Topological Response Theory of Doped Topological Insulators, Phys. Rev. Lett. 107, 206602 (2011).

[47] S. L. Adler, Axial-Vector Vertex in Spinor Electrodynamics, Phys. Rev. 177, 2426 (1969).

[48] J. S. Bell and R. Jackiw, A PCAC Puzzle: $\pi^{0} \rightarrow \gamma \gamma$ in the $\sigma$-Model, Il Nuovo Cimento A 60, 47 (1969).

[49] H. B. Nielsen and M. Ninomiya, Absence of Neutrinos on a Lattice: (I). Proof by Homotopy Theory, Nucl. Phys. B185, 20 (1981).

[50] H. B. Nielsen and M. Ninomiya, Absence of Neutrinos on a Lattice: (II). Intuitive Topological Proof, Nucl. Phys. B193, 173 (1981). 
[51] H. B. Nielsen and M. Ninomiya, The Adler-Bell-Jackiw Anomaly and Weyl Fermions in a Crystal, Phys. Lett. B 130, 389 (1983).

[52] A. A. Zyuzin and A. A. Burkov, Topological Response in Weyl Semimetals and the Chiral Anomaly, Phys. Rev. B 86, 115133 (2012).

[53] Y. Chen, S. Wu, and A. A. Burkov, Axion Response in Weyl Semimetals, Phys. Rev. B 88, 125105 (2013).

[54] V. Aji, Adler-Bell-Jackiw Anomaly in Weyl Semimetals: Application to Pyrochlore Iridates, Phys. Rev. B 85, 241101 (2012).

[55] C.-X. Liu, P. Ye, and X.-L. Qi, Chiral Gauge Field and Axial Anomaly in a Weyl Semimetal, Phys. Rev. B 87, 235306 (2013).

[56] G. Basar, D. E. Kharzeev, and H.-U. Yee, Triangle Anomaly in Weyl Semi-metals, Phys. Rev. B 89, 035142 (2014).

[57] D. T. Son and B.Z. Spivak, Chiral Anomaly and Classical Negative Magnetoresistance of Weyl Metals, Phys. Rev. B 88, 104412 (2013).

[58] K. Landsteiner, Anomaly Related Transport of Weyl Fermions for Weyl Semi-Metals, Phys. Rev. B 89, 075124 (2014).

[59] P. Goswami and S. Tewari, Axionic field theory of $(3+1)$ dimensional Weyl semimetals, Phys. Rev. B 88, 245107 (2013).

[60] A. G. Grushin, Consequences of a Condensed Matter Realization of Lorentz-Violating QED in Weyl Semi-Metals, Phys. Rev. D 86, 045001 (2012).

[61] S. T. Ramamurthy and T. L. Hughes, Patterns of Electro-Magnetic Response in Topological Semi-Metals, arXiv:1405.7377v1.

[62] B. Hetényi, DC Conductivity as a Geometric Phase, Phys. Rev. B 87, 235123 (2013).

[63] Z. Jian-Hui, J. Hua, N. Qian, and S. Jun-Ren, Topological Invariants of Metals and the Related Physical Effects, Chin. Phys. Lett. 30, 027101 (2013).

[64] H. Yao and D.-H. Lee, Topological Insulators and Topological Nonlinear $\sigma$ Models, Phys. Rev. B 82, 245117 (2010).
[65] S.-C. Zhang and J. Hu, A Four-Dimensional Generalization of the Quantum Hall Effect, Science 294, 823 (2001).

[66] B. A. Bernevig, C.-H. Chern, J.-P. Hu, N. Toumbas, and S.-C. Zhang, Effective Field Theory Description of the Higher Dimensional Quantum Hall Liquid, Ann. Phys. (Amsterdam) 300, 185 (2002).

[67] R. D. King-Smith and D. Vanderbilt, Theory of Polarization of Crystalline Solids, Phys. Rev. B 47, 1651 (1993).

[68] D. J. Thouless, Quantization of Particle Transport, Phys. Rev. B 27, 6083 (1983).

[69] K.-Y. Yang, Y.-M. Lu, and Y. Ran, Quantum Hall Effects in a Weyl Semimetal: Possible Application in Pyrochlore Iridates, Phys. Rev. B 84, 075129 (2011).

[70] G. E. Volovik, The Universe in a Helium Droplet, International Series of Monographs on Physics (Oxford University Press, Oxford, 2009).

[71] Y. Ran, Y. Zhang, and A. Vishwanath, One-Dimensional Topologically Protected Modes in Topological Insulators with Lattice Dislocations, Nat. Phys. 5, 298 (2009).

[72] T. L. Hughes, H. Yao, and X.-L. Qi, Majorana Zero Modes in Dislocations of $\mathrm{Sr}_{2} \mathrm{RuO}_{4}$, Phys. Rev. B 90, 235123 (2014).

[73] Z. Wang and S.-C. Zhang, Chiral Anomaly, Charge Density Waves, and Axion Strings from Weyl Semimetals, Phys. Rev. B 87, 161107 (2013).

[74] Y. Li, S.-C. Zhang, and C. Wu, Topological Insulators with SU(2) Landau Levels, Phys. Rev. Lett. 111, 186803 (2013).

[75] X.-G. Wen, Projective Construction of Non-Abelian Quantum Hall Liquids, Phys. Rev. B 60, 8827 (1999); B. Swingle, M. Barkeshli, J. McGreevy, and T. Senthil, Correlated Topological Insulators and the Fractional Magnetoelectric Effect, Phys. Rev. B 83, 195139 (2011); J. Maciejko, X.-L. Qi, A. Karch, and S.-C. Zhang, Fractional Topological Insulators in Three Dimensions, Phys. Rev. Lett. 105, 246809 (2010).

[76] J. K. Jain, Composite-Fermion Approach for the Fractional Quantum Hall Effect, Phys. Rev. Lett. 63, 199 (1989). 